\title{
37. GEOCHEMICAL HISTORY OF POST-JURASSIC SEDIMENTATION IN THE CENTRAL NORTHWESTERN PACIFIC, SOUTHERN HESS RISE, DEEP SEA DRILLING PROJECT SITE 465
}

\author{
I. M. Varentsov, Geological Institute of the U.S.S.R. Academy of Sciences, Moscow, U.S.S.R.
}

\begin{abstract}
Analysis of the distribution of major components and heavy metals in a section of post-Jurassic deposits of southern Hess Rise (Deep Sea Drilling Project Site 465), the results of processing analytical data by factor analysis, interpretation of information on mineralogy and lithology of sediments, and evaluation of rates of component accumulation enable distinction of the main stages in the geochemical history: (1) Late Albian-early Cenomanian (early oceanic), in which the accumulation of mostly shallow-water carbonate sediments of turbiditic nature enriched in organic matter and volcanogenic materials was proceeding; the sediments are characterized by maximally high contents of $\mathrm{Fe}, \mathrm{Mn}$, $\mathrm{SiO}_{2}, \mathrm{Al}_{2} \mathrm{O}_{3}$, and associated heavy metals, present in the form of basaltic volcaniclastic material and, to a lesser extent, hydrothermal and exhalation products and materials of their post-sedimentary transformation (smectite, hydromica, $\mathrm{Mn}-\mathrm{Fe}-\mathrm{Mg}$-carbonates); extremely high rates of accumulation of components are representative of this given stage. (2) Late Cretaceous (middle Cenomanian-late Maastrichtian), in which the accumulating sediments were considerably eroded along some hiatuses (middle Cenomanian-middle Turonian; late Coniacian; early Campanian); during the Cenomanian-Coniacian, this region is assumed to have been in the subequatorial zone of high biological productivity, within the general northward movement of the Pacific Plate (Lancelot and Larson, 1975; Lancelot, 1978; van Andel, 1974). Considering geochemical features, the sediments of this stage are similar to biogenic carbonate pelagic oozes of the open ocean. (3) Early Tertiary (early-late Paleocene), in which the beginning of the Tertiary is characterized by accumulation of carbonate nannofossil oozes admixed with siliceous remains; sedimentation was proceeding in the northern oligotrophic zone of the Pacific Ocean. (4) Tertiary-Quaternary, in which foraminifer-nannofossil pelagic oozes with geochemical features typical of these varieties were accumulated; during the Eocene-Pliocene biogenic carbonate sedimentation was repeatedly disturbed by hiatuses.

Thus, the established geochemical stages of post-Jurassic sedimentation of the region reflect, as a whole, the evolution of post-Jurassic sedimentation in the central northwestern Pacific. It is noteworthy that although the timing of events of these stages (for the Cretaceous particularly) for certain regions (Mid-Pacific Mountains, Nauru Basin, etc.) are essentially different, the general tendency of geochemical evolution of the basins is common.
\end{abstract}

\section{INTRODUCTION}

Hess Rise is among the largest aseismic structures of the central northwestern Pacific. It is located much higher than the surrounding abyssal areas of the Pacific Plate. Such disposition of the rise allows us to believe that a relatively complete section of biogenic sediments can be developed in this rather shallow-water region (see Site 465 report, this volume).

This paper is a continuation of the series of works on the geochemical history of post-Jurassic sedimentation in the central northwestern Pacific, based on sediments penetrated during DSDP Leg 62 (see papers by Varentsov et al., on Sites 463 and 464, this volume).

As with the previous works, the objective of this study is to elucidate the main features of the geochemical history of sedimentation in the southern region of Hess Rise, recorded in the chemical and mineral composition of sediments, on the basis of study of the geochemistry of the major components, heavy metals, trace elements, and data on mineralogy and lithology.

It should be emphasized that particular attention is focused in this work on studying the local features of the geochemical history of sedimentation in the given

\footnotetext{
${ }^{1}$ Initial Reports of the Deep Sea Drilling Project, Volume 62
}

structural area, and their relationships with the general evolution of geochemistry of post-Jurassic sedimentation in the central northwestern Pacific.

\section{MATERIALS AND METHODS}

This work was based on the data of chemical and mineral composition of sediments penetrated by Holes 465 and $465 \mathrm{~A}$. The studies were carried out at the Geological Institute of the U.S.S.R. Academy of Sciences, Moscow. The results of lithologic-mineralogical investigations of sediments are presented in other chapters of this volume.

A detailed description of methodological features of the studies was given in our paper on Site 463 (Varentsov et al., this volume).

It should be noted that the determination of chemical components of sediments was carried out at the Geological Institute of the U.S.S.R. Academy of Sciences: major components by methods of bulk analysis, heavy metals by optical emission spectroscopy, with the use of international standards (Zolotarev and Choporov, 1978; Kirkpatrick, 1979).

In order to exclude the diluting influence of carbonate and siliceous components (and terrigenous components being present), and to reduce the composition of sediments to a geochemically comparable basis, the chemical analyses were recalculated to a clastic-free, silicafree, carbonate-free basis (Varentsov and Blazhchishin, 1976).

The analytical data were processed by a computer (EC-1022) (D. A. Kazimirov, P. K. Ryabushkin), following the program of factor analysis (R- and Q-mode; Davis, 1973; Harman, 1967).

The determinations used in interpreting the data of chemical analysis of clay minerals in the $<0.001-\mathrm{mm}$ fraction are given in accordance with the data by Rateev et al. (this volume). In addition, all chemically analyzed samples were studied as thin sections under the microscope, and by diffractometry methods, with necessary treatment for elucidation of their general composition (natural specimens). 


\section{PARAGENETIC ASSOCIATIONS OF COMPONENTS}

Paragenetic associations of chemical components were identified through the results of processing of analytical data by the factor analysis method. Two types of determinations were studied: (1) the data of the chemical analyses presented in weight percent (air-dry); (2) data recalculated to terrigenous-free, silica-free, carbonate-free. The established paragenetic groupings were interpreted against the background of data on mineralogy and lithology of sediments, and the possible geochemical conditions of their formation.

\section{DATA OF CHEMICAL ANALYSIS (TABLES 1, 2, 3; FIG. 1)}

Assemblage $\mathrm{IA}(+)$ : $\mathrm{SiO}_{2}(0.39)-\mathrm{Al}_{2} \mathrm{O}_{3}(0.59)-\mathrm{MgO}$ $(0.55)-\mathrm{C}_{\text {org }}(0.75)-\mathrm{Fe}(0.12)-\mathrm{P}(0.49)-\mathrm{Cr}(0.48)-\mathrm{Ni}$ $(0.60)-\mathrm{V}(\mathbf{0 . 9 0 )}-\mathrm{Cu}(0.81)-\mathrm{Mo}(\mathbf{0 . 8 7})$. This assemblage is represented by aluminosilicate components in the form of basaltic volcaniclastics, considerably transformed into montmorillonite-illite minerals. The association is characterized by closely related organic carbon, $\mathrm{P}$, and a number of heavy metals: $\mathrm{Cr}, \mathrm{Ni}, \mathrm{V}, \mathrm{Cu}, \mathrm{Mo}$.

The distribution of this assemblage of components is rather limited in the section (Fig. 1): late Albian-early Cenomanian laminated limestones essentially enriched in basaltic volcaniclastic material and dispersed sapropel-like organic matter.

Assemblage IB (-): $\mathrm{CaO}(-0.23)-\mathrm{Na}_{2} \mathrm{O}(-0.77)-$ $\mathrm{CO}_{2}(-\mathbf{0 . 1 7})$. This assemblage is presented by calcium carbonate in the form of unconsolidated biogenic remains: nannofossils, foraminifers, micrite. As a rule, this almost unaltered calcium carbonate is closely related to $\mathrm{Na}$ taken up from sea water. Noteworthy is a distinct stratigraphic localization of this assemblage in the section (Fig. 1): nannofossil and foraminifer-nannofossil oozes of the Pleistocene/late Campanian.

Assemblage IIA (+): $\mathrm{SiO}_{2}(0.65)-\mathrm{Al}_{2} \mathrm{O}_{3}(0.60)-\mathrm{Na}_{2} \mathrm{O}$ $(0.32)-\mathrm{K}_{2} \mathrm{O}(0.74)-\mathrm{Fe}(0.73)-\mathrm{Mn}(0.41)-\mathrm{P}(0.63)-\mathrm{Ni}$ (0.40)-V (0.30)-Cu (0.17)-Ga (0.32)-Mo (0.28). This is an aluminosilicate phase represented mostly by mixedlayer minerals of the montmorillonite-illite type, closely related to $\mathrm{P}$ and a set of heavy metals: Ni, V, Ga, Mo.

The predominant development of this association within late Albian laminated limestones (e.g., Samples 29-1, 81-82 cm; 37-2, 74-75 cm), appreciably enriched in fine basaltic volcaniclastic material, can be interpreted as an evidence that the given group of components is the products of alteration (hydromicatization, smectitization) of volcanogenic components.

Assemblage IIB (-): $\mathrm{CaO}(-0.91)-\mathrm{CO}_{2}(-0.88)-$ $\mathrm{C}_{\text {org }}(\mathbf{- 0 . 1 8})-\mathrm{Co}(\mathbf{- 0 . 5 2 )}$. This assemblage is represented by calcium carbonate (main phase), $\mathrm{C}_{\text {org }}$, and $\mathrm{Co}$, which are slightly related to the main phase.

The assemblage is developed mostly in the lower part of Unit I (lower than Sample 9-4, 105-107 cm; depth $120.55 \mathrm{~m}$ ), and among the rocks of Unit II (laminated limestones) (Fig. 1).

Study of thin sections and correlation to the discussed assemblage IB ( - ) enables us to consider group IIB ( -$)$ mostly the product of recrystallization of the initial biogenic calcium carbonate in the course of its epigenetic transformation. This conclusion agrees with the data on determination of density (see Site 465 report, this volume): for Unit I below Core $10(134-276 \mathrm{~m})$, the average density is $1.60 \pm 0.02 \mathrm{~g} / \mathrm{cm}^{3}$; for Unit II $(276-411.7 \mathrm{~m})$ it is $2.22 \mathrm{~g} / \mathrm{cm}^{3}$; whereas for the higher horizons $(0-135 \mathrm{~m})$ the average density is $1.54 \pm 0.04$ $\mathrm{g} / \mathrm{cm}^{3}$. However, the process of recrystallization of biogenic carbonate proceeds unevenly (the interval 120.55$134.0 \mathrm{~m}$ may be regarded as a zone of intermediate den-

Table 1. Chemical composition of Cenozoic and Mesozoic sediments of the central northwestern Pacific, southern Hess Rise, DSDP Site 465 (\%, wt, air-dry weight portion).

\begin{tabular}{|c|c|c|c|c|c|c|c|c|c|c|c|c|c|c|c|c|c|c|c|c|c|c|c|}
\hline \multirow{3}{*}{$\begin{array}{c}\text { Sample } \\
\text { (interval in } \mathrm{cm} \text { ) }\end{array}$} & \multicolumn{14}{|c|}{ Component } & \multirow{2}{*}{\multicolumn{9}{|c|}{ (wt. ${ }^{5} \cdot 10^{-4}$ ) }} \\
\hline & \multicolumn{14}{|c|}{ (wt. $\%$, air-dry) } & & & & & & & & & \\
\hline & $\mathrm{SiO}_{2}$ & $\mathrm{Al}_{2} \mathrm{O}_{3}$ & $\mathrm{Fe}_{2} \mathrm{O}_{3}$ & $\mathrm{CaO}$ & $\mathrm{MgO}$ & $\mathrm{MnO}$ & $\mathrm{Na}_{2} \mathrm{O}$ & $\mathrm{K}_{2} \mathrm{O}$ & $\mathrm{CO}_{2}$ & $\mathrm{C}_{\text {org }}$ & $\mathrm{P}_{2} \mathrm{O}_{5}$ & $\mathrm{Fe}_{\text {tot }}$ & $\mathrm{Mn}_{\text {tot }}$ & $P_{\text {tot }}$ & $\mathrm{Cr}$ & $\mathrm{Ni}$ & $\mathrm{v}$ & $\mathrm{Cu}$ & Co & $\mathrm{Pb}$ & $\mathrm{Ga}$ & $\mathrm{Ge}$ & Mo \\
\hline $465-2-1,108-110$ & 5.75 & 1.57 & 0.74 & 49.46 & 0.42 & 0.01 & 1.28 & 0.51 & 38.95 & - & 0.07 & 0.52 & 0.01 & 0.03 & $<10$ & $<10$ & $<15$ & $<15$ & $<10$ & $<10$ & $<5$ & $<1$ & $<1.5$ \\
\hline $3-2,110-112$ & 0.44 & 0.01 & 0.15 & 54.84 & 0.41 & 0.02 & 1.08 & 0.15 & 41.75 & - & 0.07 & 0.10 & 0.01 & 0.03 & $<10$ & $<10$ & $<15$ & $<15$ & $<10$ & $<10$ & $<5$ & $<1$ & $<1.5$ \\
\hline $4-2,10-12$ & 0.26 & 0.05 & 0.06 & 54.92 & 0.42 & 0.01 & 1.08 & 0.15 & 43.45 & - & 0.05 & 0.04 & 0.01 & 0.04 & $<10$ & $<10$ & $<15$ & $<15$ & $<10$ & $<10$ & $<5$ & $<1$ & $<1.5$ \\
\hline $5-2,70-72$ & 1.45 & 0.16 & 0.06 & 53.78 & 0.22 & 0.06 & 1.35 & 0.20 & 41.35 & - & 0.14 & 0.04 & 0.05 & 0.06 & $<10$ & $<10$ & $<15$ & $<15$ & $<10$ & $<10$ & $<5$ & $<i$ & $<1.5$ \\
\hline $465 \mathrm{~A}-1-1,104-106$ & 2.52 & 0.10 & 0.20 & 52.51 & 0.07 & 0.04 & 1.45 & 0.31 & 40.60 & - & 0.10 & 0.14 & 0.03 & 0.04 & $<10$ & $<10$ & $<15$ & $<15$ & $<10$ & $<10$ & $<5$ & $<1$ & $<1.5$ \\
\hline $465-6 \cdot 2,98-100$ & 0.57 & 0.05 & 0.19 & 54.07 & - & 0.04 & 1.27 & 0.25 & 42.35 & - & 0.09 & 0.13 & 0.03 & 0.04 & $<10$ & $<10$ & $<15$ & $<15$ & $<10$ & $<10$ & $<5$ & $<1$ & $<1.5$ \\
\hline $465 \mathrm{~A}-3-1,118-120$ & 0.72 & 0.10 & 0.20 & 52.84 & - & 0.02 & 0.54 & 0.25 & 42.30 & - & 0.10 & 0.14 & 0.02 & 0.04 & $<10$ & $<10$ & $<15$ & $<15$ & $<10$ & $<10$ & $<5$ & $<1$ & $<1.5$ \\
\hline $465-10-5,93-95$ & 2.61 & 0.16 & 0.20 & 52.64 & 0.09 & 0.01 & 1.18 & 0.25 & 40.55 & - & 0.03 & 0.14 & 0.01 & 0.01 & $<10$ & $<10$ & $<15$ & $<15$ & $<10$ & $<10$ & $<5$ & $<i$ & $<1,5$ \\
\hline $465 \mathrm{~A}-9-4,105-107$ & 0.06 & 0.05 & 0.07 & 53.88 & - & 0.01 & 1.64 & 0.20 & 42.20 & - & 0.004 & 0.05 & 0.01 & 0.002 & $<10$ & $<10$ & $<15$ & $<15$ & $<10$ & $<10$ & $<5$ & $<1$ & $<1.5$ \\
\hline $10-1,64-68$ & - & - & 0.35 & 53.96 & - & 0.01 & 1.45 & 0.83 & 42.35 & - & 0.004 & 0.24 & 0.01 & 0.002 & $<10$ & $<10$ & $<15$ & $<15$ & $<10$ & $<10$ & $<5$ & $<1$ & $<1.5$ \\
\hline $11-1,26-30$ & 0.91 & 0.02 & 0.20 & 52.74 & - & 0.02 & 1.45 & 0.83 & 41.80 & - & 0.03 & 0.14 & 0.02 & 0.01 & $<10$ & $<10$ & $<15$ & $<$ is & $<10$ & $<10$ & $<5$ & $<i$ & $<1.5$ \\
\hline $12-1,70-74$ & 0.42 & 0.08 & 0.20 & 54.39 & - & 0.01 & 0.77 & 0.14 & 42.85 & - & - & 0.14 & 0.01 & - & $<10$ & $<10$ & $<15$ & $<15$ & $<10$ & $<10$ & $<5$ & $<1$ & $<1.5$ \\
\hline $15-1,120-122$ & 0.30 & 0.08 & 0.20 & 54.20 & 0.34 & 0.02 & 1.06 & 0.14 & 42.15 & - & 0.04 & 0.14 & 0.02 & 0.02 & $<10$ & $<10$ & $<15$ & $<$ is & $<10$ & $<10$ & $<5$ & $<1$ & $<1.5$ \\
\hline $16-4,80-82$ & 0.32 & 0.06 & 0.52 & 54.21 & 0.17 & 0.02 & 1.06 & 0.09 & 40.25 & - & 0.02 & 0.36 & 0.02 & 0.01 & $<10$ & $<10$ & $<15$ & $<15$ & $<10$ & $<10$ & $<5$ & $<i$ & $<1.5$ \\
\hline $17-1,140-142$ & - & 0.02 & 0.20 & 54.18 & 0.52 & 0.02 & 1.06 & 0.09 & 41.55 & - & - & 0.14 & 0.02 & - & $<10$ & $<10$ & $<15$ & $<20$ & $<10$ & $<10$ & $<5$ & $<1$ & $<1.5$ \\
\hline $18-1,136-138$ & 0.32 & 0.06 & 0.13 & 54.06 & 0.26 & 0.01 & 1.06 & 0.14 & 42.95 & - & 0.02 & 0.09 & 0.01 & 0.01 & $<10$ & $<10$ & $<15$ & $<20$ & $<10$ & $<10$ & $<5$ & $<1$ & $<1.5$ \\
\hline $19-2,146-148$ & 0.70 & 0.01 & 0.13 & 53.80 & 0.35 & 0.01 & 1.16 & 0.09 & 41.60 & - & 0.03 & 0.09 & 0.01 & 0.01 & $<10$ & $<10$ & $<15$ & $<20$ & $<10$ & $<10$ & $<5$ & $<1$ & $<1.5$ \\
\hline $20-1,94-96$ & 0.08 & 0.06 & 0.13 & 54.36 & 0.34 & 0.01 & 1.06 & 0.09 & 41.85 & - & 0.03 & 0.09 & 0.01 & 0.01 & $<10$ & $<10$ & $<15$ & $<20$ & $<10$ & $<10$ & $<5$ & $<1$ & $<1.5$ \\
\hline $26-1,57-58$ & 0.46 & 0.31 & 0.13 & 53.87 & 0.44 & 0.01 & 0.53 & 0.15 & 42.45 & 0.88 & 0.04 & 0.09 & 0.01 & 0.02 & 10 & $<10$ & 49 & $<20$ & $<10$ & $<10$ & $<5$ & $<1$ & 2,8 \\
\hline $27-1,76-77$ & 0.66 & 0.04 & 0.37 & 53.58 & 0.26 & 0.01 & 0.53 & 0.10 & 41.80 & 0.83 & 0.08 & 0.26 & 0.01 & 0.03 & II & 11 & 35 & $<20$ & $<10$ & $<10$ & $<5$ & $<1$ & 2.8 \\
\hline $28-1,81-82$ & 3.02 & 0.83 & 0.35 & 48.62 & 0.18 & 0.02 & 0.68 & 0.31 & 37.60 & 1,93 & 0.14 & 0.24 & 0.02 & 0.06 & is & 12 & 72 & $<20$ & $<10$ & $<10$ & $<5$ & $<1$ & 3.7 \\
\hline $29-1,81-82$ & 17.67 & 6.13 & 3.58 & 36.70 & 1.23 & 0.05 & 1.52 & 0.61 & 27.10 & 0.03 & 0.73 & 2.50 & 0.04 & 0.32 & $<10$ & $<10$ & 41 & $<20$ & $<10$ & $<10$ & $<5$ & $<1$ & 2.1 \\
\hline $30-1,70-71$ & 0.54 & 0.12 & 0.22 & 54.00 & 0.35 & 0.01 & 0.53 & 0.10 & 42.35 & 0.71 & 0.04 & 0.15 & 0.01 & 0.02 & $<10$ & $<10$ & 56 & $<20$ & $<10$ & $<10$ & $<5$ & $<1$ & 2.1 \\
\hline $32-1,58-59$ & 0.98 & 0.26 & 0.13 & 53.88 & 0.18 & 0.01 & 0.53 & 0.20 & 41.95 & 0.57 & 0.03 & 0.09 & 0.01 & 0.0 & $<10$ & $<10$ & 59 & $<20$ & $<10$ & $<10$ & $<5$ & $<1$ & 3.1 \\
\hline $33-1,15-16$ & 1.63 & 0.53 & 0.22 & 52.71 & 0.35 & 0.01 & 0.60 & 0.15 & 41.25 & 1.15 & 0.07 & 0.15 & 0.01 & 0.03 & $<10$ & $<10$ & 66 & $<20$ & $<10$ & $<10$ & $<5$ & $<1$ & 4.3 \\
\hline $34-1,41-42$ & 0.62 & 0.28 & 0.13 & 54.19 & 0.18 & - & 0.53 & 0.15 & 42.55 & 0.65 & 0.06 & 0.09 & - & 0.03 & $<10$ & $<10$ & 58 & $<20$ & $<10$ & $<10$ & $<5$ & $<i$ & 5.1 \\
\hline $36-2,92-93$ & 1.95 & 0.57 & 0.26 & 50.76 & 0.44 & 0.01 & 0.60 & 0.25 & 40.00 & 1.12 & 0.18 & 0.18 & 0.01 & 0.08 & $<10$ & 20 & 260 & $<20$ & $<10$ & $<10$ & $<5$ & $<1$ & 9.9 \\
\hline $37-2,74-75$ & 4.36 & 1.31 & 0.33 & 46.39 & 0.35 & 0.01 & 0.76 & 0.51 & 35.85 & - & $\left\{\begin{array}{c}0.25 \\
0.33\}\end{array}\right\}$ & 0.23 & 0.01 & $\left\{\begin{array}{l}0.11 \\
0.14\end{array}\right.$ & 13 & 49 & $>500$ & 30 & $<10$ & $<10$ & $<5$ & $<1$ & 16.2 \\
\hline 38 & 3.7 & 0 & & & 0.44 & 0.0 & 0.8 & 0.4 & & - & $\begin{array}{c}(0.33)) \\
0.30\end{array}$ & & & $\begin{array}{c}(0.14) \\
0.13\end{array}$ & $<10$ & 38 & 2 & $=20$ & & & $<5$ & $<1$ & 16 \\
\hline $39-1,113-114$ & 2.24 & 0.56 & 0.26 & 50.15 & 0.44 & 0.01 & 0.85 & 0.31 & 39.40 & 2.42 & 0.23 & 0.1 & 0.0 & 0.10 & $<10$ & 15 & 210 & $<20$ & $<10$ & $<10$ & $<5$ & $<1$ & 7.3 \\
\hline $40-2,42-43$ & 2.59 & 0.85 & 0.55 & 51.35 & 0.71 & 0.13 & 0.68 & 0.31 & 40.10 & 0.54 & 0.10 & 0.38 & 0.10 & 0.04 & $<10$ & $<10$ & 155 & $<20$ & $<10$ & $<10$ & $<5$ & $<i$ & 5.8 \\
\hline
\end{tabular}


Table 2. Results of factor analysis (R-mode) for chemical components (wt. \%), Cenozoic and Mesozoic sediments of the northwestern $\mathrm{Pa}$ cific, DSDP Site 465.

\begin{tabular}{ccrrr}
\hline & & \multicolumn{3}{c}{$\begin{array}{c}\text { Factor Loadings } \\
\text { (after rotation) }\end{array}$} \\
\cline { 3 - 5 } No. & Component & \multicolumn{1}{c}{$\mathrm{I}$} & \multicolumn{1}{c}{ II } & \multicolumn{1}{c}{ III } \\
\hline 1 & $\mathrm{SiO}_{2}$ & 0.39 & 0.65 & \\
2 & $\mathrm{Al}_{2} \mathrm{O}_{3}$ & 0.59 & 0.60 & 0.32 \\
3 & $\mathrm{CaO}_{4}$ & -0.23 & -0.91 & \\
4 & $\mathrm{MgO}$ & 0.55 & & 0.51 \\
5 & $\mathrm{Na}_{2} \mathrm{O}$ & -0.77 & 0.32 & \\
6 & $\mathrm{~K}_{2} \mathrm{O}$ & -0.07 & 0.74 & 0.19 \\
7 & $\mathrm{CO} 2$ & -0.17 & -0.88 & \\
8 & $\mathrm{C}_{2}$ org & 0.75 & -0.18 & \\
9 & $\mathrm{Fe}$ & 0.12 & 0.73 & \\
10 & $\mathrm{Mn}$ & & 0.41 & -0.51 \\
11 & $\mathrm{P}$ & 0.49 & 0.63 & \\
12 & $\mathrm{Cr}$ & 0.48 & & \\
13 & $\mathrm{Ni}$ & 0.60 & 0.40 & 0.38 \\
14 & $\mathrm{~V}$ & 0.90 & 0.30 & \\
15 & $\mathrm{Cu}$ & 0.81 & 0.17 & \\
16 & $\mathrm{Co}$ & & 0.52 & -0.47 \\
17 & $\mathrm{~Pb}$ & & & 0.77 \\
18 & $\mathrm{Ga}$ & & 0.32 & -0.34 \\
19 & $\mathrm{Ge}$ & & & \\
20 & $\mathrm{Mo}$ & 0.87 & 0.28 & \\
Dispersion input $(\%)$ & 34.75 & 17.06 & 9.66 \\
Total dispersion (\%) & 34.75 & 51.81 & 61.48 \\
\hline & & & & \\
\hline
\end{tabular}

Table 3. Stratigraphic distribution of factor scores (R-mode) for chemical components (wt. \%) in Cenozoic and Mesozoic sediments of the northwestern Pacific, DSDP Site 465.

\begin{tabular}{rclrrr}
\hline & & & \multicolumn{3}{c}{$\begin{array}{c}\text { Factor scores } \\
\text { (after rotation) }\end{array}$} \\
\cline { 4 - 6 } No. & $\begin{array}{c}\text { Sample } \\
\text { (interval in cm) }\end{array}$ & Stratigraphy & \multicolumn{1}{c}{ I } & \multicolumn{1}{c}{ II } & \multicolumn{1}{c}{ III } \\
\hline & & & & \\
1 & $465-2-1,108-110$ & Pleistocene & -0.63 & 0.97 & -1.34 \\
2 & $3-2,110-112$ & U. Paleocene & -0.76 & -0.27 & 0.74 \\
3 & $4-2,10-12$ & U. Paleocene & -0.47 & -0.86 & -0.20 \\
4 & $5-2,70-72$ & U. Paleocene & -0.83 & 0.01 & -1.68 \\
5 & 465 A-1-1, 104-106 & U. Paleocene & -1.00 & 0.43 & -1.23 \\
6 & $465-6-2,98-100$ & U. Paleocene & -1.29 & 0.42 & 0.91 \\
7 & 465 A-3-1, 118-120 & L. Paleocene & -0.71 & 0.31 & 0.83 \\
8 & $465-10-5,93-95$ & U. Maastrichtian & -0.75 & 0.10 & 0.03 \\
9 & 465 A-9-4, 105-107 & L. Maastrichtian & -1.08 & -0.64 & 1.23 \\
10 & $10-1,64-68$ & L. Maastrichtian & -1.37 & -0.24 & 1.32 \\
11 & $11-1,26-30$ & L. Maastrichtian & -1.50 & 0.85 & 1.33 \\
12 & $12-1,70-74$ & L. Maastrichtian & -0.53 & -0.58 & 0.78 \\
13 & $15-1,120-122$ & U. Campanian & -0.64 & -0.38 & -1.30 \\
14 & $16-1,80-82$ & U. Campanian & -0.75 & -0.38 & -0.54 \\
15 & $17-1,140-142$ & U. Campanian & -0.41 & -0.78 & -0.06 \\
16 & $18-1,136-138$ & U. Campanian & -0.41 & -0.31 & 1.04 \\
17 & $19-2,146-148$ & U. Campanian & -0.31 & -0.67 & 0.12 \\
18 & $20-1,94-96$ & U. Campanian & 0.26 & -1.02 & -0.90 \\
19 & $26-1,57-58$ & U. Cenomanian & 0.96 & -0.87 & -0.23 \\
20 & $27-1,76-77$ & U. Cenomanian & 0.85 & -0.71 & -0.10 \\
21 & $28-1,81-82$ & U. Albian & 1.23 & 0.65 & -0.12 \\
22 & $29-1,81-82$ & U. Albian & -0.47 & 3.81 & -0.95 \\
23 & $30-1,70-71$ & U. Albian & 0.99 & -1.12 & 0.29 \\
24 & $32-1,58-59$ & U. Albian & 0.87 & -0.88 & -0.04 \\
25 & $33-1,15-16$ & U. Albian & 1.02 & -0.55 & -1.25 \\
26 & $34-1,41-42$ & U. Albian & 1.19 & -1.00 & 0.53 \\
27 & $36-2,92-93$ & U. Albian & 1.67 & 0.11 & 0.19 \\
28 & $37-2,74-75$ & U. Albian & 1.92 & 1.88 & 2.06 \\
29 & $38-1,73-74$ & U. Albian & 1.36 & 1.12 & 0.87 \\
30 & $39-1,113-114$ & U. Albian & 1.38 & 0.14 & -0.13 \\
31 & $40-2,42-43$ & U. Albian & 0.72 & 0.45 & -2.20 \\
\hline & & & & & \\
\hline & & & &
\end{tabular}

sities). The admixture of clay components and organic matter inhibits the process of recrystallization, especially in the lower parts of Unit II (see Fig. 1).

Assemblage IIIA (+): $\mathrm{K}_{2} \mathrm{O}(-0.19)-\mathrm{Ni}(0.38)-\mathrm{Pb}$ (0.77). This assemblage is composed of hydromica and $\mathrm{K}$-feldspar components rich in nickel and lead.

It is noteworthy that the intervals of well-pronounced development of this assemblage are observed in the lower half of early Albian deposits (see Fig. 1) and in early Maastrichtian sediments.

These deposits are characterized by noticeable enrichment in clay components developed after basic volcaniclastic material. In this case, in early Albian rocks the maximum content of basaltic volcaniclastic components (Assemblage IA; Fig. 1) corresponds to the maximum of the cluster concerned.

Assemblage IIIB (-): $\mathrm{Al}_{2} \mathrm{O}_{3}(-32)-\mathrm{MgO}(-0.51)-$ Mn (-0.51)-Co $(-0.47)-G a(-0.34)$. The real phase composition of this assemblage is not clear. Study of thin sections of rocks and sediments enables us to suppose that this assemblage is represented by manganese hydroxides associated with authigenic magnesian smectites and heavy metals ( $\mathrm{Co}, \mathrm{Ga})$.

This assemblage is best pronounced near the contact with basalts, the boundary between Units II and III, where considerable exhalation-hydrothermal influence took place (Sample 465A-40-2,42-43 cm). At the other levels of the section, this assemblage is composed of products of deep alteration of basaltic volcaniclastic material (see Fig. 1).

\section{DATA OF CHEMICAL ANALYSES RECALCULATED (TABLES 4-6; FIG. 2)}

Assemblage IA (+): $\mathrm{CaO}(0.24)-\mathrm{Fe}(0.30)-\mathrm{Mn}(0.13)$. This cluster is represented by surplus amounts of lime, iron, and to a lesser extent manganese. The most appreciable values of factor loadings of this assemblage correspond to the similar parameters of the above-considered Assemblage IIA (Fig. 1); this enables us to interpret such a cluster of components as evidence of the presence of a specific calcium-iron smectite phase-a product of alteration of basaltic volcaniclastic material. Such an interpretation does not contradict the available data on mineralogy.

Assemblage IB (-): $\mathrm{Na}_{2} \mathrm{O}(-0.41)-\mathrm{K}_{2} \mathrm{O}(-0.80)-\mathrm{Cr}$ $(-0.90)-\mathrm{Ni}(-0.89)-\mathrm{V}(-0.78)-\mathrm{Cu}(-0.96)-\mathrm{Co}$ $(-0.89)-\mathrm{Pb}(-0.89)-\mathrm{Ga}(-0.94)-\mathrm{Ge}(-0.80)-\mathrm{Mo}$ (-0.67). This group of components plays the role of a diluting agency in the general paragenetic association with the preceding Assemblage IA (+). If we assume that both Assemblages IA (+) and IB (-) are represented by mixed-layer minerals (montmorillonite/illite; see also assemblage IIA (+); Fig. 1), the given association of components (IB (+); Fig. 2) reflects the distribution of illite packets proper and related heavy metals in the section. The predominant development of associations in upper Albian-lower Cenomanian rocks enriched with basaltic volcaniclastic material is indirect evidence of its epigenetic origin. 


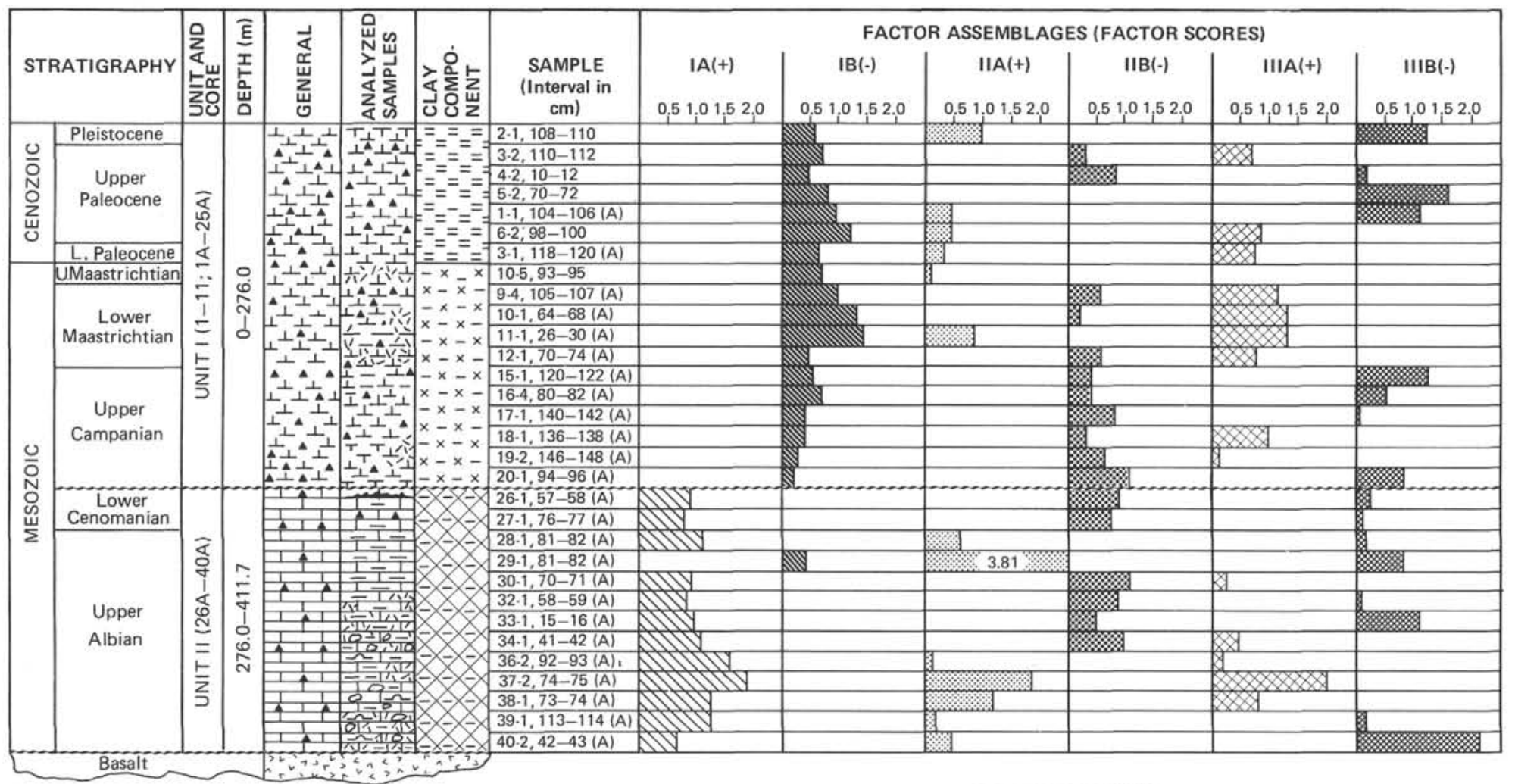

FACTOR LOADINGS

CLAY COMPONENTS

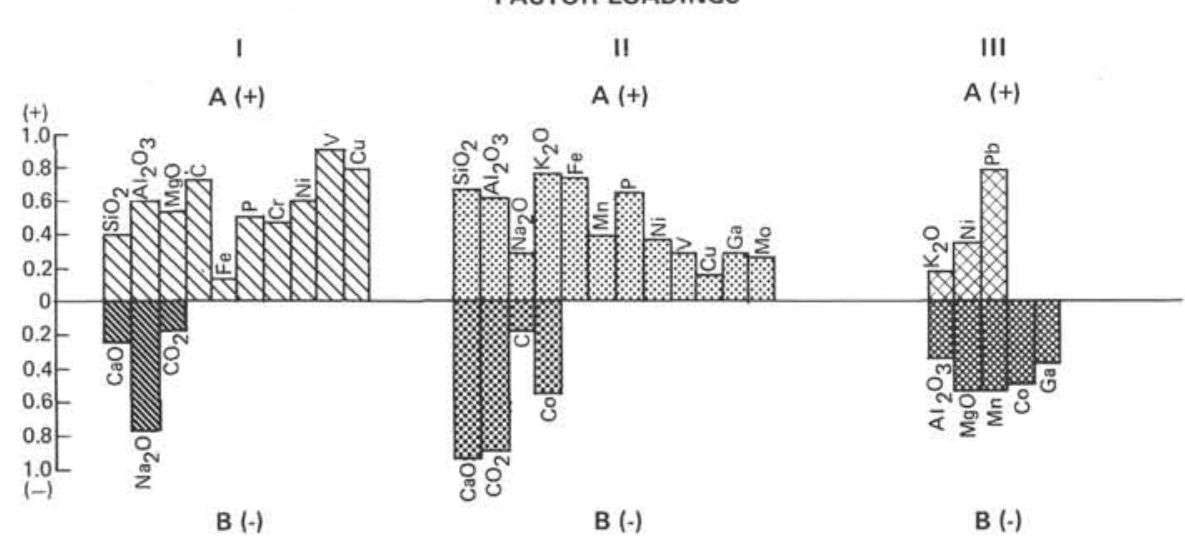

Figure 1. Stratigraphic distribution of factor scores of the main paragenetic assemblages of chemical components in the section of post-Jurassic deposits of the central northwestern Pacific, southern Hess Rise, DSDP Site 465 (data of chemical analysis recalculated to air-dry weight). Lithologic symbols are those used in DSDP Initial Reports. 
Table 4. Chemical composition of Cenozoic and Mesozoic sediments of the central northwestern Pacific, southern Hess Rise, DSDP Site 465. (wt. $\%$ recalculated to terrigenous-free, silica-free, carbonate-free).

\begin{tabular}{|c|c|c|c|c|c|c|c|c|c|c|c|c|c|c|c|c|}
\hline $\begin{array}{c}\text { Sample } \\
\text { (interval in cm) }\end{array}$ & $\mathrm{CaO}$ & $\mathrm{MgO}$ & $\mathrm{Na}_{2} \mathrm{O}$ & $\mathrm{K}_{2} \mathrm{O}$ & $\mathrm{Fe}_{\text {tot }}$ & $\mathrm{Mn}_{\text {tot }}$ & $P_{\text {tot }}$ & $\mathrm{Cr}$ & $\mathrm{Ni}$ & V & $\mathrm{Cu}$ & Co & $\mathrm{Pb}$ & $\mathrm{Ga}$ & $\mathrm{Ge}$ & Mo \\
\hline $65-2-1,108-110$ & - & 13.182 & 65.316 & 15.775 & 4.214 & - & 1.297 & 0.011 & 0.011 & 0.027 & 0.054 & 0.043 & 0.043 & 0.016 & 0.004 & 0.007 \\
\hline $3-2,110-112$ & 47.695 & 11.997 & 31.665 & 4.371 & 2.875 & 0.292 & 0.879 & 0.029 & 0.029 & 0.044 & 0.044 & 0.029 & 0.029 & 0.015 & 0.003 & 0.004 \\
\hline $4-2,10-12$ & - & 8.645 & 77.661 & 10.302 & - & - & 2.867 & 0.070 & 0.071 & 0.081 & 0.107 & 0.072 & 0.072 & 0.035 & 0.007 & 0.011 \\
\hline $5-2,70-72$ & 36.219 & 6.975 & 46.371 & 6.146 & 0.449 & 1.554 & 2.051 & 0.031 & 0.031 & 0.048 & 0.034 & 0.034 & 0.034 & 0.017 & 0.003 & 0.005 \\
\hline $465 \mathrm{~A}-1-1,104-106$ & 27.132 & 2.149 & 52.662 & 10.780 & 4.480 & 1.082 & 1.442 & 0.035 & 0.035 & 0.052 & 0.053 & 0.036 & 0.036 & 0.017 & 0.004 & 0.005 \\
\hline $465-6-2,98-100$ & 4.682 & - & 70.671 & 13.543 & 6.800 & 1.661 & 2.218 & 0.054 & 0.055 & 0.082 & 0.083 & 0.055 & 0.055 & 0.027 & 0.006 & 0.008 \\
\hline $465 \mathrm{~A}-3-1,118-120$ & - & - & 65.437 & 28.812 & - & - & 4.835 & 0.116 & 0.116 & 0.175 & 0.179 & 0.121 & 0.121 & 0.059 & 0.012 & 0.018 \\
\hline $465-10-5,93-95$ & 36.532 & 2.834 & 46.177 & 8.976 & 4.448 & 0.374 & 0.370 & 0.035 & 0.035 & 0.055 & 0.057 & 0.039 & 0.039 & 0.019 & 0.004 & 0.006 \\
\hline $465 \mathrm{~A}-9-4,105-107$ & 4.299 & - & 82.843 & 9.761 & 2.124 & 0.496 & 0.091 & 0.049 & 0.050 & 0.074 & 0.075 & 0.050 & 0.050 & 0.025 & 0.005 & 0.008 \\
\hline $10-1,64-68$ & - & - & 57.454 & 32.888 & 9.272 & - & 0.079 & 0.040 & 0.040 & 0.059 & 0.059 & 0.040 & 0.040 & 0.020 & 0.004 & 0.006 \\
\hline $11-1,26-30$ & - & - & 63.176 & 36.057 & - & - & 0.432 & 0.043 & 0.043 & 0.065 & 0.065 & 0.043 & 0.043 & 0.022 & 0.004 & 0.007 \\
\hline $12-1,70-74$ & - & - & 84.869 & 14.293 & - & - & - & 0.106 & 0.106 & 0.161 & 0.164 & 0.110 & 0.110 & 0.054 & 0.011 & 0.017 \\
\hline $15-1,120-122$ & 21.552 & 15.374 & 40.049 & 5.992 & 5.852 & 0.915 & 0.915 & 0.045 & 0.045 & 0.067 & 0.068 & 0.046 & 0.046 & 0.023 & 0.005 & 0.007 \\
\hline $16-4,80-82$ & 63.176 & 3.553 & 23.0 & 1.788 & 7.630 & 0.432 & 0.214 & 0.0 & 0.021 & 0.032 & 0.032 & 0.022 & 0.022 & 0.011 & 0.002 & 0.003 \\
\hline $17-1,140-142$ & 39.992 & 16.994 & 34.743 & 2.854 & 4.495 & 0.653 & - & 0.0 & 0.032 & 0.049 & 0.065 & 0.0 & 0.033 & 0.016 & 0.003 & 0.005 \\
\hline $18-1,136-138$ & - & - & 87.580 & 10. & - & - & 0.812 & 0.080 & 0.080 & & 0.1 & 0.0 & 0.0 & 0.041 & 0.008 & 0.012 \\
\hline $19-2,146-148$ & 31.195 & 14.012 & 46. & & 3.533 & 0.400 & 0.400 & 0.040 & 0.040 & & & & & 0 . & 0. & 0.006 \\
\hline $20-1,94-96$ & 39.001 & 12.859 & 40.816 & 3.166 & 3.089 & 0.378 & 0.378 & 0.037 & 0.037 & 0.056 & 0.076 & 0. & & & 0. & 06 \\
\hline $26-1,57-58$ & - & 32.048 & 53.690 & 11.133 & - & - & 1.977 & 0.083 & 0.094 & 0.489 & 0.198 & 0.101 & 0.1 & & 0.010 & 0.029 \\
\hline $27-1,76-77$ & 20.092 & 17.319 & 35.719 & 6.359 & 17.116 & 0.670 & 2.016 & 0.073 & 0.073 & 0.235 & 0.135 & 0.067 & 0.067 & 0.033 & 0.007 & 0.019 \\
\hline $28-1,81-82$ & 33.011 & 5.241 & 38.734 & 11.747 & 6.024 & 1.024 & 3.434 & 0.066 & 0.048 & 0.404 & 0.108 & 0.054 & 0.054 & 0.024 & 0.005 & 0.022 \\
\hline $29-1,81-82$ & 22.958 & 11.727 & 26.867 & - & 31.618 & 0.410 & 6.393 & - & - & 0.002 & 0.004 & 0.009 & 0.009 & - & - & 0.002 \\
\hline $30-1,70-71$ & 0.179 & 30.139 & 46.953 & 7.423 & 11.626 & 0.859 & 1.744 & 0.080 & 0.080 & 0.492 & 0.176 & 0.089 & 0.089 & 0.043 & 0.009 & C.019 \\
\hline $32-1,58-59$ & 28.681 & 11.833 & 40.592 & 12.852 & 3.605 & 0.705 & 0.705 & 0. & 0.070 & 0.447 & 0.149 & 0.076 & 0.076 & 0.036 & 0.078 & 0.024 \\
\hline $33-1,15-16$ & 3.666 & 26.668 & 52.785 & 6.965 & 5.498 & 0.733 & & & 0.064 & & 0.1 & 0. & 0.082 & 0.037 & 0.008 & 0.038 \\
\hline $34-1,41-42$ & - & 18.142 & 62.949 & 13.515 & 365 & - & & & & & & & 0. & 0.056 & 0.012 & 0.062 \\
\hline $36-2,92-93$ & - & 14.655 & 57.224 & 17.048 & - & - & 7.776 & 0.070 & 0.169 & & & & & 0.040 & 0.009 & 0.098 \\
\hline $37-2,74-75$ & 24.72 & 10.819 & 37.359 & 17.480 & 0.480 & 0.320 & 5.596 & 0.032 & 0.229 & 2.617 & 0.139 & 0.048 & 0.048 & 0.016 & 0.005 & 0.086 \\
\hline $38-1,73-74$ & - & - & 61.903 & 25.634 & $\div$ & - & 9.641 & 0.038 & 0.260 & 2.097 & 0.130 & 0.069 & 0.069 & 0.031 & 0.007 & 0.122 \\
\hline $39-1,113-114$ & - & 23.736 & 51.942 & 14.607 & 1.826 & - & 6.170 & 0.044 & 0.076 & 1.297 & 0.113 & 0.057 & 0.057 & 0.025 & 0.006 & 0.045 \\
\hline $40-2,42-43$ & 4.614 & 31.886 & 33.286 & 9.955 & 12.236 & 5.029 & 1.918 & 0.031 & 0.076 & 0.773 & 0.093 & 0.047 & 0.057 & 0.021 & 0.005 & 0.030 \\
\hline
\end{tabular}

Table 5. Results of factor analysis (R-mode) of chemical components (wt. \%, recalculated to clastic-free, carbonate-free, silica-free), Cenozoic and Mesozoic sediments of the northwestern Pacific, DSDP Site 465.

\begin{tabular}{ccrrr}
\hline & & \multicolumn{3}{c}{$\begin{array}{c}\text { Factor Loadings } \\
\text { (after rotation) }\end{array}$} \\
\cline { 3 - 5 } No. & Components & \multicolumn{1}{c}{$\mathrm{I}$} & \multicolumn{1}{c}{ II } & \multicolumn{1}{c}{ III } \\
\hline 1 & $\mathrm{CaO}$ & 0.24 & 0.91 & -0.16 \\
2 & $\mathrm{MgO}$ & & 0.62 & 0.49 \\
3 & $\mathrm{Na} 2 \mathrm{O}$ & -0.41 & -0.73 & \\
4 & $\mathrm{~K} 2 \mathrm{O}$ & -0.80 & & \\
5 & $\mathrm{Fe}$ & 0.30 & 0.80 & \\
6 & $\mathrm{Mn}$ & 0.13 & 0.92 & -0.14 \\
7 & $\mathrm{P}$ & & -0.04 & 0.82 \\
8 & $\mathrm{Cr}$ & -0.94 & & -0.16 \\
9 & $\mathrm{Ni}$ & -0.89 & & \\
10 & $\mathrm{~V}$ & -0.78 & -0.05 & 0.53 \\
11 & $\mathrm{Cu}$ & -0.96 & & \\
12 & $\mathrm{Co}$ & -0.89 & -0.36 & \\
13 & $\mathrm{~Pb}$ & -0.89 & -0.36 & \\
14 & $\mathrm{Ga}$ & -0.94 & & \\
15 & $\mathrm{Ge}$ & -0.80 & & \\
16 & $\mathrm{Mo}$ & -0.67 & -0.20 & 0.63 \\
Dispersion input (\%) & 58.25 & 15.56 & 9.82 \\
Total dispersion (\%) & 58.25 & 73.81 & 83.63 \\
\hline
\end{tabular}

Table 6. Stratigraphic distribution of factor scores (R-mode) for chemical components (wt. \%, recalculated to clastic-free, carbonate-free, silica-free), in Cenozoic and Mesozoic sediments, central northwestern Pacific, DSDP Site 465.

\begin{tabular}{|c|c|c|c|c|c|}
\hline \multirow[b]{2}{*}{ No. } & \multirow{2}{*}{$\begin{array}{l}\text { Sample } \\
\text { (interval in } \mathrm{cm} \text { ) }\end{array}$} & \multirow[b]{2}{*}{ Stratigraphy } & \multicolumn{3}{|c|}{$\begin{array}{c}\text { Factor scores } \\
\text { (after rotation) }\end{array}$} \\
\hline & & & I & II & III \\
\hline 1 & $465-2-1,108-118$ & Pleistocene & 1.08 & -1.00 & 0.18 \\
\hline 2 & $3-2,110-112$ & Upper Paleocene & 0.68 & 0.78 & -0.22 \\
\hline 3 & $4-2,10-12$ & Upper Paleocene & -0.02 & -1.32 & 0.26 \\
\hline 4 & $5-2,70-72$ & Upper Paleocene & 0.60 & 0.52 & -0.23 \\
\hline 5 & $465 \mathrm{~A}-1-1,104-106$ & Upper Paleocene & 0.33 & 0.58 & -0.52 \\
\hline 6 & $465-6-2,98-100$ & Upper Paleocene & -0.23 & 0.15 & -0.99 \\
\hline 7 & $465 \mathrm{~A}-3-1,118-120$ & Lower Paleocene & -0.76 & -1.46 & -0.12 \\
\hline 8 & $465-10-5,93-95$ & Upper Maastrichtian & 0.24 & 0.63 & -0.67 \\
\hline 9 & $465 A-9-4,105-107$ & Lower Maastrichtian & -0.12 & -0.11 & -1.68 \\
\hline 10 & $10-1,64-68$ & Lower Maastrichtian & 0.47 & -0.99 & -1.27 \\
\hline 11 & $11-1,26-30$ & Lower Maastrichtian & 0.57 & -1.69 & -0.75 \\
\hline 12 & $12-1,70-74$ & Lower Maastrichtian & -0.91 & -1.41 & -2.12 \\
\hline 13 & $15-1,120-122$ & Upper Campanian & -0.02 & 0.79 & -0.37 \\
\hline 14 & $16-4,80-82$ & Upper Campanian & 1.11 & 0.95 & -0.47 \\
\hline 15 & $17-1,140-142$ & Upper Campanian & 0.23 & 1.06 & -1.74 \\
\hline 16 & $18-1,136-138$ & Upper Campanian & -0.27 & -1.70 & -0.63 \\
\hline 17 & $19-2,146-148$ & Upper Campanian & 0.17 & 0.71 & -0.49 \\
\hline 18 & $20-1,94-96$ & Upper Campanian & 0.24 & 0.77 & -0.43 \\
\hline 19 & $26-1,57-58$ & Lower Cenomanian & -0.65 & -0.86 & 0.91 \\
\hline 20 & $27-1,76-77$ & Lower Cenomanian & -0.69 & 1.18 & 0.29 \\
\hline 21 & $28-1,81-82$ & Upper Albian & -0.43 & 1.02 & 0.45 \\
\hline 22 & $29-1,81-82$ & Upper Albian & 4.36 & -0.07 & 1.32 \\
\hline 23 & $30-1,70-71$ & Upper Albian & -0.96 & 0.83 & 0.33 \\
\hline 24 & $32-1,58-59$ & Upper Albian & -1.32 & 1.28 & 0.08 \\
\hline 25 & $33-1,15-16$ & Upper Albian & -0.85 & 0.82 & 0.61 \\
\hline 26 & $34-1,41-42$ & Upper Albian & -1.02 & -0.53 & 1.03 \\
\hline 27 & $36-2,92-93$ & Upper Albian & -0.69 & -0.93 & 1.82 \\
\hline 28 & $37-2,74-75$ & Upper Albian & -0.49 & 0.81 & 1.62 \\
\hline 29 & $38-1,73-74$ & Upper Albian & -0.31 & -1.52 & 1.40 \\
\hline 30 & $39-1,113-114$ & Upper Albian & -0.10 & -0.50 & 1.55 \\
\hline 31 & $40-2,42-43$ & Upper Albian & -0.21 & 1.21 & 0.85 \\
\hline
\end{tabular}




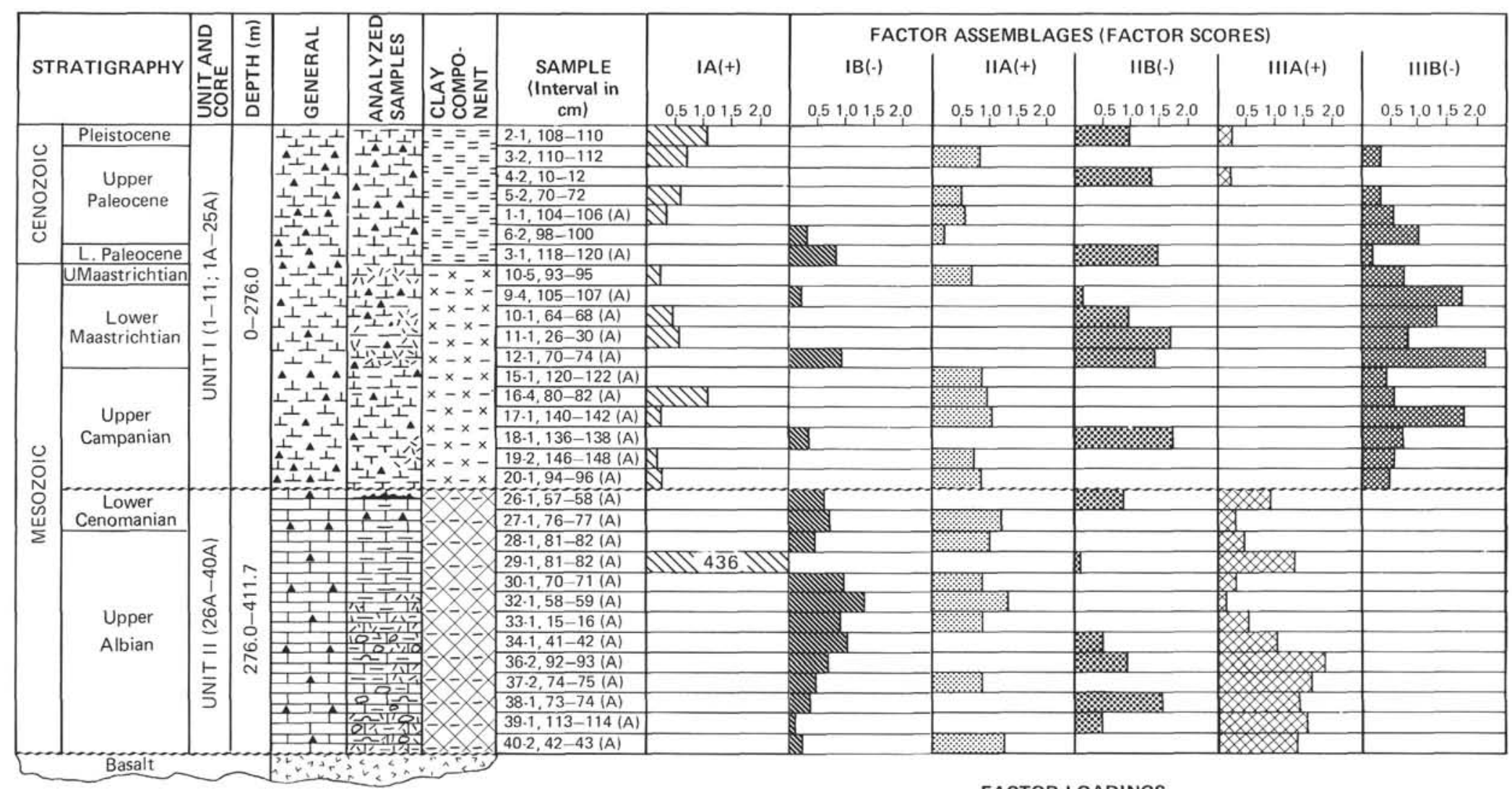

FACTOR LOADINGS

\section{CLAY COMPONENTS}

$\bar{F}_{\bar{*}}=\mathrm{P}$ Polymineral assemblage of illite, chlorite,

$\bar{F}=\overline{ }=$ and a small admixture of montmorillonite. An assemblage represented by $\mathrm{Fe}(\mathrm{Al})$.

$x-x-$ montmorillonite, with a slight admixture

$-x-x$ in some interbeds. Representative is the

presence of zeolite (heulandite) and cherty

segregates composed of crystobalite-ridymite.

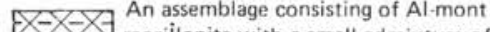

- - morillonite with a small admixture of illite.

quartz is present too.

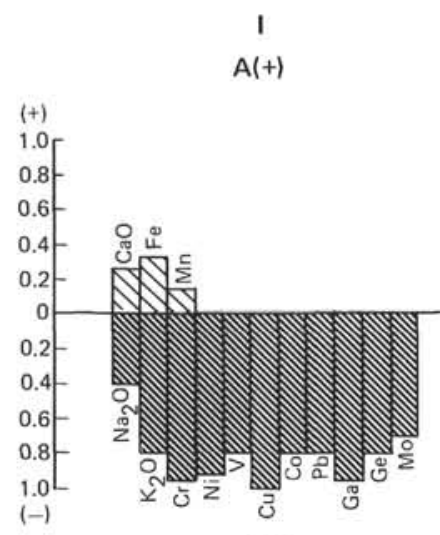

$B(-)$
II

$A(+)$

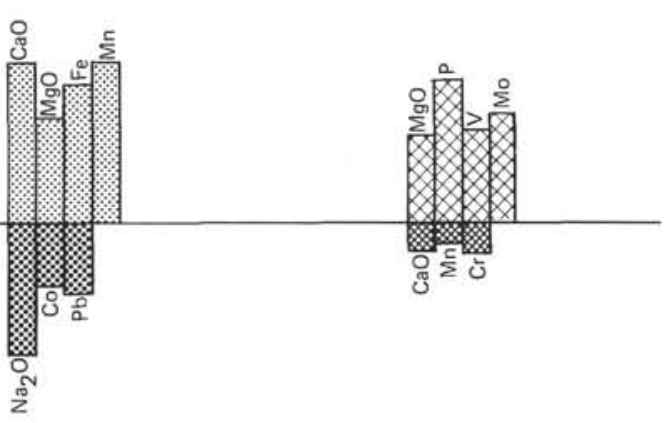

B(-)

Figure 2. Stratigraphic distribution of factor scores of the main paragenetic associations of chemical components (recalculated to terrigenous-free, carbonate-free, silicafree) in the section of post-Jurassic deposits of the central northwestern Pacific southern Hess Rise, DSDP Site 465. Symbols as in Figure 1. 
Assemblage IIA (+): $\mathrm{CaO}(0.91)-\mathrm{MgO}(0.62)-\mathrm{Fe}$ $(0.80)-M n(0.92)$. This assemblage is represented by profoundly altered basaltic volcaniclastic material and related segregates, crusts, and coatings of $\mathrm{Mn}$ - and $\mathrm{Fe}-$ hydroxides. The assemblage is developed mostly in upper Albian-lower Cenomanian and upper Campanian sediments.

Assemblage IIB (-): $\mathrm{Na}_{2} \mathrm{O}(0.73)-\mathrm{Co}(-36)-\mathrm{Pb}$ $(-\mathbf{0 . 3 6})$. This assemblage of components is negatively correlated with Assemblage IIA (+). One can assume that Assemblage IIB (-) is represented by volcanogenic components relatively rich in $\mathrm{Na}_{2} \mathrm{O}$, and that $\mathrm{Co}$ and $\mathrm{P}$ are rather moderately associated with these components. Elimination of the diluting effect of carbonate and siliceous components enables us to establish a rather even distribution of these two assemblages in the section.

Assemblage IIIA (+): MgO (0.49)-P (0.82)-V (0.53)-Mo (0.63). This assemblage is represented by a set of excessive (against the accepted norm) components; their distribution is clearly confined to upper $\mathrm{Al}$ bian-lower Cenomanian rocks (Fig. 2). The similar distribution in the section, as mentioned above, is representative of assemblage IA (+) (Fig. 1). Thus, the identity of mineral composition of these two assemblages is evident: basaltic volcaniclastic material transformed into montmorillonite-illite minerals and associated heavy metals.

Assemblage IIIB (-): $\mathrm{CaO}(-0.16)-\mathrm{Mn}(-0.14)-\mathrm{Cr}$ (-0.16). The slight significance of factor loadings enables us to assess the composition of the assemblage rather tentatively. Worthy of attention is the clear localization of this assemblage in the section: Unit I (upper Campanian-Pleistocene). A similar stratigraphic distribution is characteristic of the above-considered assemblage IB (-) (Fig. 1), represented by biogenic carbonates (nannofossil oozes and foraminifer-nannofossil oozes).

It should be emphasized that Assemblage IIIB (-) is composed of a set of components that are excessive relative to normative carbonate molecules. The identity of distribution of both assemblages and peculiarities of the composition of components allows us to think that Assemblage IIIB (-) may be represented by remnant phases after recrystallization of biogenic carbonates, diagenetic Mn-hydroxides in particular. The reality of such post-sedimentary recrystallization of biogenic carbonate is evidenced by the results of study of the sediments under a microscope, and the features of distribution of paragenetic groups (IB (-), IIB (-); Fig. 1) in the section.

\section{AVERAGE CONTENTS OF COMPONENTS AND RATES OF THEIR ACCUMULATION}

(TABLES 1, 4, 7; FIGS. 3-5)

\section{Distribution of Average Contents}

Analysis of the specific features of distribution of average contents of the major components of sediments and rocks in the main geochronological subdivisions of the section (Tables 1, 4; Figs. 3, 4), with allowance for the above-mentioned forms of their occurrences (see Figs. 1, 2), enables us to outline the following stages and phases in the geochemical history of sedimentation:

Late Albian-early Cenomanian (early oceanic). This phase is characterized by relatively high concentrations of $\mathrm{SiO}_{2}, \mathrm{Al}_{2} \mathrm{O}_{3}, \mathrm{Fe}, \mathrm{Mn}, \mathrm{P}$, and associated heavy metals, and high contents of organic carbon (Late Albian, $0.0-2.42$, avg. 0.83 ); early Cenomanian, $0.83-0.88$, avg. 0.86 ). Two phases can be defined in the stage concerned: (1) a late Albian phase in which the sediments are characterized by relatively high $\mathrm{SiO}_{2}, \mathrm{Al}_{2} \mathrm{O}_{3}, \mathrm{Fe}$, $\mathrm{Mn}$, and $\mathrm{P}$ contents, and associated heavy metals; enrichment in these components is related to the considerable amounts of volcaniclastic components of basaltic composition, and to hydrothermal and exhalative products; (2) an early Cenomanian phase, in which the lithologic features and chemistry of major components $\left(\mathrm{CaCO}_{3}, \mathrm{C}_{\text {org }}\right.$, etc. $)$ in the sediments are similar to the late Albian; nevertheless, an appreciable decrease in concentrations of the components of volcanogenic origin $\left(\mathrm{SiO}_{2}, \mathrm{Al}_{2} \mathrm{O}_{3}, \mathrm{Fe}, \mathrm{Mn}, \mathrm{P}\right)$ and heavy metals is observed.

Late Cretaceous (middle Cenomanian-late Maastrichtian). The chemical composition of sediments of the initial phases of this stage (middle CenomanianSantonian) remains obscure because of the hiatuses and poor core recovery, and the predominance of cherts in this part of the section. However, fragmentary data of the shipboard descriptions (see Site 465 report, this volume) enable us to believe that the sediments of this stage are formationally homogeneous.

The contents of major components and associated heavy metals in sediments of the later period of this stage (late Campanian-late Maastrichtian) do not exceed the values characteristic of pelagic foraminifernannofossil oozes of the open ocean. Noteworthy is a somewhat higher content of normative molecules of $\mathrm{MnCO}_{3}, \mathrm{FeCO}_{3}$, and $\mathrm{MgCO}_{3}$ in early Campanian-early Maastrichtian sediments, due to epigenetic alteration of an admixture of mafic volcaniclastic material.

Early Tertiary (early-late Paleocene). Essential predominance of $\mathrm{CaCO}_{3}$ in the form of foraminifernannofossil oozes considerably masks the features of authigenic components recognized while analyzing the recalculated data; for instance, $\mathrm{P}$ and $\mathrm{K}_{2} \mathrm{O}$ concentrations (early Paleocene; Fig. 4); noteworthy are high concentrations of the normative molecules $\mathrm{MnCO}_{3}$ and $\mathrm{FeCO}_{3}$ (early Paleocene) and $\mathrm{MgCO}_{3}$ (late Paleocene) (Fig. 3).

Tertiary-Quaternary. A sharp decrease of thickness in this stage and their redeposited character (EocenePliocene sediments) resulted in very restricted chemicalanalysis data. The information of Pleistocene sediments (see Tables 1, 4, 7; Figs. 3, 4) shows that they are represented by foraminifer-nannofossil oozes with an admixture of basaltic volcaniclastic material. Such volcanogenic components resulted in relatively high contents of 
Table 7. Average contents and mean rates of accumulation of chemical components for the main geochronological subdivisions of post-Jurassic sediments, central northwestern Pacific, southern Hess Rise, DSDP Site 465.

\begin{tabular}{|c|c|c|c|c|c|c|c|c|c|c|c|}
\hline Unit & Lithology & Cores & $\begin{array}{l}\text { Sub-bottom } \\
\text { Depth } \\
\text { (m) }\end{array}$ & $\begin{array}{l}\text { Thickness } \\
\text { (m) }\end{array}$ & $\begin{array}{c}\text { Stage } \\
\text { (substage) }\end{array}$ & Cores & $\begin{array}{l}\text { Sub-bottom } \\
\text { Depth } \\
\text { (m) }\end{array}$ & $\begin{array}{c}\text { Thickness } \\
\text { (m) }\end{array}$ & $\begin{array}{l}\text { Density } \\
\left(\mathrm{g} / \mathrm{cm}^{3}\right)\end{array}$ & $\begin{array}{c}\text { Water } \\
\text { Content } \\
(\%)\end{array}$ & $\begin{array}{l}\text { Duration } \\
\text { (m.y.) }\end{array}$ \\
\hline \multirow{6}{*}{ I } & \multirow{6}{*}{$\begin{array}{l}\text { Nannofossil, } \\
\text { foraminifer- } \\
\text { nannofossil } \\
\text { ooze }\end{array}$} & \multirow{6}{*}{$1-11,1 \mathrm{~A}-25 \mathrm{~A}$} & \multirow{6}{*}{$0-276$} & \multirow{6}{*}{276.0} & Pleistocene & 0 to $2-1,130 \mathrm{~cm}$ & $0.0-2.3$ & 2.3 & 1.54 & $40.0(?)$ & 1.8 \\
\hline & & & & & U. Paleocene & $2-6,60 \mathrm{~cm}$ to $6, \mathrm{CC}$ & $9.1-48.5$ & 39.4 & 1.54 & 35.0 & 6.5 \\
\hline & & & & & L. Paleocene & $7-1$ to $8, C C$ & $48.5-61.5$ & 13.0 & 1.54 & $35.0(?)$ & 5.0 \\
\hline & & & & & U. Maastricht. & $9-1$ to $8 \mathrm{~A}, \mathrm{CC}$ & $61.5-106.0$ & 43.5 & 1.54 & 35.0 & 2.5 \\
\hline & & & & & L. Maastricht. & $9-1 \mathrm{~A}$ to 12 & $106.0-144.5$ & 38.5 & 1.54 & 35.1 & 2.5 \\
\hline & & & & & $\begin{array}{l}\text { U. Campanian } \\
\text { U. Turonian }\end{array}$ & $12-1$ to $20, \mathrm{CC}$ & $144.5-229.0$ & 84.5 & 1.60 & 34.8 & 4.0 \\
\hline \multirow[t]{2}{*}{ II } & Laminated & $26 \mathrm{~A}-40 \mathrm{~A}$ & $276.0-411.7$ & 135.7 & L. Cenoman. & $25, \mathrm{CC}$ to $27, \mathrm{CC}$ & $267.0-295.5$ & 28.5 & 2.22 & 5.60 & 2.5 \\
\hline & limestone & & & & U. Albian & $28-1,40, \mathrm{CC}$ & $295.5-419.5$ & 123.5 & 2.22 & 12.7 & 4.0 \\
\hline III & Trachyte & $40 A-46 A$ & $411.7-476.0$ & 64.3 & & & & & & & \\
\hline
\end{tabular}

$\mathrm{Al}_{2} \mathrm{O}_{3}$ and $\mathrm{Fe}$, and in post-sedimentary dissolution in appreciable amounts (see Fig. 3) of normative molecules of $\mathrm{FeCO}_{3}$ and $\mathrm{MnCO}_{3}$.

\section{Rates of Component Accumulation (Table 7; Fig. 5)}

Methodological aspects of calculation of average linear rates of sedimentation, accumulation of components, selection of chronostratigraphic scales, and duration of the main chronostratigraphic intervals are given in our work on the geochemical history of sedimentation at Site 463 (Varentsov et al., this volume).

Analysis of the recalculated data on average linear rates of sedimentation and accumulation of components and the values of their average contents and forms of occurrence in the sediment permitted definition of stages of geochemical history of sedimentation of the region. On the whole, these stages and phases correspond to the intervals established in the analysis of distribution of average contents of the components. It is important to emphasize that hiatuses in the lower part of the section (middle Cenomanian-middle Turonian; late Coniacian; early Campanian; Fig. 5) result in the final analysis in decrease of already-minimal values of rates of sediment and component accumulation. Thus, interpretation of these data can be carried out only within the context of information on geochemistry, lithology, mineralogy, and sedimentation conditions.

Late Albian - early Cenomanian (early oceanic). This stage is characterized by maximum accumulation rates of sediments and components. Noteworthy are rather high rates of accumulation of components supplied in the form of volcaniclastic material of basaltic composition, and products of hydrothermal exhalations: $\mathrm{Fe}$, $\mathrm{Mn}, \mathrm{Al}_{2} \mathrm{O}_{3}, \mathrm{SiO}_{2}, \mathrm{P}$, and heavy metals (Bender et al., 1971; Boström, 1973; MacArthur and Elderfield, 1977). Sediments of turbiditic nature accumulated under conditions of a relatively shallow-water stagnant basin (deficiency of oxygen in bottom water).

For the late Albian phase of this stage, the rates of sedimentation and rates of accumulation of components representative of the initial (proto-oceanic) stages of development of a basin have been established (Tiercelin and Faure, 1978); in particular, they are close to parameters for the late Barremian-early Aptian phase in the western part of the Mid-Pacific Mountains, Site 463 (Varentsov et al., this volume).

For the early Cenomanian phase of the stage, the values of rates of sedimentation and accumulation of components are appreciably lower than those for the late Albian (Table 7; Fig. 5). This may be related to a considerable extent to the presence of a lower middle Cenomanian-middle Turonian hiatus, and to a lesser extent to a lower rate of sedimentation with the deepening of the basin.

Late Cretaceous (middle Cenomanian-late Maastrichtian). The given geochronological intervals are presented by deposits of drastically decreased thicknesses, as a result of hiatuses (Fig. 5). This is confirmed by the fact that the calculated average rates of sedimentation and accumulation of components do not correspond with the rates that might have been expected in the absence of hiatuses during the northward movement of the Pacific Plate at that time. According to the modifications of the horizontal movement of the Pacific Plate, the southern region of Hess Rise should have crossed the equatorial zone of high biological productivity either during the Cenomanian-Coniacian (Lancelot and Larson, 1975; Lancelot, 1978), or near that interval of time (van Andel, 1974). The available data show that during the entire post-Jurassic the position of the region under study was higher than the CCD.

It is known that the northern and southern boundaries of the recent near-equatorial zone of high biological productivity of the Pacific Ocean are approximately within $10^{\circ} \mathrm{N}$ and $10^{\circ} \mathrm{S}$. It is assumed that during the early Maastrichtian the southern region of Hess Rise had not yet moved beyond the northern boundary of the equatorial zone of high biological productivity. The crossing of the boundary likely took place in the late Maastrichtian, as evidenced by the continued high values of rates of sedimentation and accumulation of components (Table 7; Fig. 5).

Along with this interpretation, the possibility should be taken into consideration that essential increasing of thickness (i.e., rates of accumulation) of the early Maastrichtian sediments may have been caused by local contribution and redeposition of sedimentary material from tectonic uplift of nearby structural blocks (details 
Table 7. (Continued).

\begin{tabular}{|c|c|c|c|c|c|c|c|c|c|c|c|c|c|}
\hline \multirow[b]{3}{*}{$\left(\mathrm{mm} \cdot 10^{-3} \cdot \mathrm{ur}^{-1}\right)$} & \multirow[b]{3}{*}{$\left(\mathrm{mg} \cdot \mathrm{cm}^{-2} \cdot 10^{-3} \cdot \mathrm{yr}^{-1}\right)$} & \multicolumn{12}{|c|}{$\begin{array}{l}\text { Average Contents of Components (wt. } \% \text { ) and Rate of Accumulation } \\
\qquad\left(\mathrm{mg} \cdot \mathrm{cm}^{-2} \cdot 10^{-3} \cdot \mathrm{yr}^{-1}\right)\end{array}$} \\
\hline & & \multicolumn{2}{|c|}{$\mathrm{SiO}_{2}$} & \multicolumn{2}{|c|}{$\mathrm{Al}_{2} \mathrm{O}_{3}$} & \multicolumn{2}{|c|}{$\mathrm{CaCO}_{3}$} & \multicolumn{2}{|c|}{$\mathrm{Fe}$} & \multicolumn{2}{|c|}{ Mn } & \multicolumn{2}{|c|}{ P } \\
\hline & & $(\%)$ & $\begin{array}{l}\text { Accum. } \\
\text { Rate }\end{array}$ & $(\%)$ & $\begin{array}{l}\text { Acc. } \\
\text { Rate }\end{array}$ & $(\%)$ & $\begin{array}{c}\text { Accum. } \\
\text { Rate }\end{array}$ & $(\%)$ & $\begin{array}{l}\text { Acc. } \\
\text { Rate }\end{array}$ & $(\%)$ & $\begin{array}{l}\text { Acc. } \\
\text { Rate }\end{array}$ & $\begin{array}{l}\text { Acc. } \\
(\%)\end{array}$ & Rate \\
\hline 1.28 & 146 & 5.75 & 8.4 & 1.57 & 2.3 & 88.27 & 128.9 & 0.52 & 0.8 & 0.01 & 0.01 & 0.03 & 0.04 \\
\hline 6.1 & 726 & 1.05 & 7.6 & 0.07 & 0.5 & 95.14 & 690.7 & 0.09 & 0.7 & 0.03 & 0.20 & 0.04 & 0.29 \\
\hline 2.6 & 309 & 0.72 & 2.2 & 0.10 & 0.3 & 94.30 & 291.4 & 0.14 & 0.4 & 0.02 & 0.06 & 0.04 & 0.12 \\
\hline 17.4 & 2070 & 2.61 & 54.0 & 0.16 & 3.3 & 92.23 & 1910.0 & 0.14 & 2.9 & 0.01 & 0.21 & 0.01 & 0.21 \\
\hline 15.4 & 1520 & 0.35 & 5.3 & 0.04 & 0.6 & 95.87 & 1457.0 & 0.14 & 2.1 & 0.01 & 0.15 & 0.003 & 0.05 \\
\hline 21.2 & 2645 & 0.29 & 7.7 & 0.05 & 1.3 & 94.70 & 2550.0 & 0.15 & 4.0 & 0.01 & 0.26 & 0.01 & 0.26 \\
\hline 11.4 & 2470 & 0.56 & 13.9 & 0.17 & 4.2 & 95.61 & 2390.0 & 0.17 & 4.2 & 0.01 & 0.25 & 0.02 & 0.50 \\
\hline 30.9 & 6467 & 3.58 & 231.5 & 1.12 & 72.4 & 88.07 & 5695.5 & 0.40 & 25.9 & 0.02 & 1.30 & 0.08 & 5.17 \\
\hline
\end{tabular}

on the structural position of the site are given in the Site 465 report, this volume).

Early Tertiary (early-late Paleocene). The beginning of the Cenozoic is characterized by a considerable decrease in rates of foraminifer-nannofossil-ooze sedimentation in the northern oligotrophic zone of the $\mathrm{Pa}$ cific Ocean. For the major components and associated heavy metals (Table 7; Fig. 5), the accumulation rates do not exceed the values characteristic of carbonate pelagic oozes (Arrhenius, 1963, 1967; Bezrukov and Romankevich, 1970; Bogdanov and Chekhovskikh, 1970; Lisitsin, 1974, 1978; MacArthur and Elderfield, 1977). Worthy of attention, however, are relatively higher values of $\mathrm{Mn}$ and $\mathrm{P}$ accumulation for the late Paleocene (mg $\left.\cdot \mathrm{cm}^{-2} \cdot 10^{-3} \mathrm{yr}^{-1}\right): \mathrm{Mn}, 0.20 ; \mathrm{P}, 0.29$. This can be due to the presence of appreciable amounts (up to 10-15\%) of an admixture of hyalopelitic, silty material of basaltic composition, essentially transformed into smectite-illite, considerably diluted with carbonate: $\mathrm{CaCO}_{3}$ to $95.14 \%$ (see Figs. 1, 2).

Late Tertiary-Quaternary. A considerable part of this period, as mentioned above, was rather poorly recovered. Thicknesses of Eocene-Pliocene deposits are sharply decreased, because of numerous hiatuses accompanied by partial redeposition and washing of sediments (see Site 465 report, this volume).

Relatively limited information on late Pliocene and Pleistocene sediments (Table 7; Fig. 5) enables us to think that foraminifer-nannofossil oozes with typical rates of component accumulation were deposited at that time (Table 7; Fig. 5).

\section{GEOCHEMICAL HISTORY OF SEDIMENTATION}

The geochemical history of post-Jurassic sedimentation of southern Hess Rise can be subdivided into stages and phases that correspond considerably to geochronological intervals established by interpretation of average contents and rates of component accumulation, studied within the general context of data on geochemistry, mineralogy, and lithology of sediments.

Late Albian-early Cenomanian (early oceanic). Representing this time are thinly-laminated, olive-gray limestones, with subordinate interbeds of gray limestones accumulated in the southern region of Hess Rise (see Site 465 report, this volume). These deposits are characterized by appreciable amounts of sapropel-like organic matter, and a significant admixture of basaltic volcaniclastic material.

Geochemical features of these sediments were described above (Figs. 1-4). They are characterized by the highest contents of $\mathrm{SiO}_{2}, \mathrm{Al}_{2} \mathrm{O}_{3}, \mathrm{Fe}, \mathrm{Mn}, \mathrm{P}$, and associated heavy metals, which were contributed with basaltic volcaniclastic material, and to a lesser extent with hydrothermal-exhalative activity, especially in the early intervals (the first half of the late Albian).

Accumulation of sediments containing fragments of shallow-water mollusks, reef-building fossils, and fragments of volcanic edifices and rocks (basalts, trachybasalts, etc.) took plate in the initial phase (late Albian), in a relatively shallow-water, apparently depressiontype basin, with stagnant bottom waters, characterized by notable hydrodynamic activity. The sediments have distinct features of turbidites. The basin was bounded by island volcanoes, reef banks, etc.

Accumulation of sediments in such a rapidly subsiding basin was characterized by rather high rates of accumulation of major components and associated metals (Fig. 5; Table 7). Such high rates of accumulation are due to three factors: (1) high rates of sedimentation in the shallow-water, progressively subsiding basin; (2) an active volcanic influence that proved responsible for relatively high contents of some major components (e.g., $\mathrm{Fe}, \mathrm{Mn}, \mathrm{Al}_{2} \mathrm{O}_{3}, \mathrm{SiO}_{2}$ ) and associated heavy metals, and supply of considerable amounts of basaltic volcaniclastic material, and lesser amounts of hydrothermal exhalation products; (3) intensive biological productivity of the planktonic zone of the basin as a result of the position of the region near the equator at that time, and the contribution of nutrient mineral components from volcanic sources. High biological productivity of this shallow basin resulted in accumulation of considerable amounts of sapropelic organic matter in carbonate sediments.

Thus, the initial (early oceanic) stage of development of the southern region of Hess Rise is characterized by well-pronounced geochemical features. A similar geochemical stage can also be observed in other regions (for 


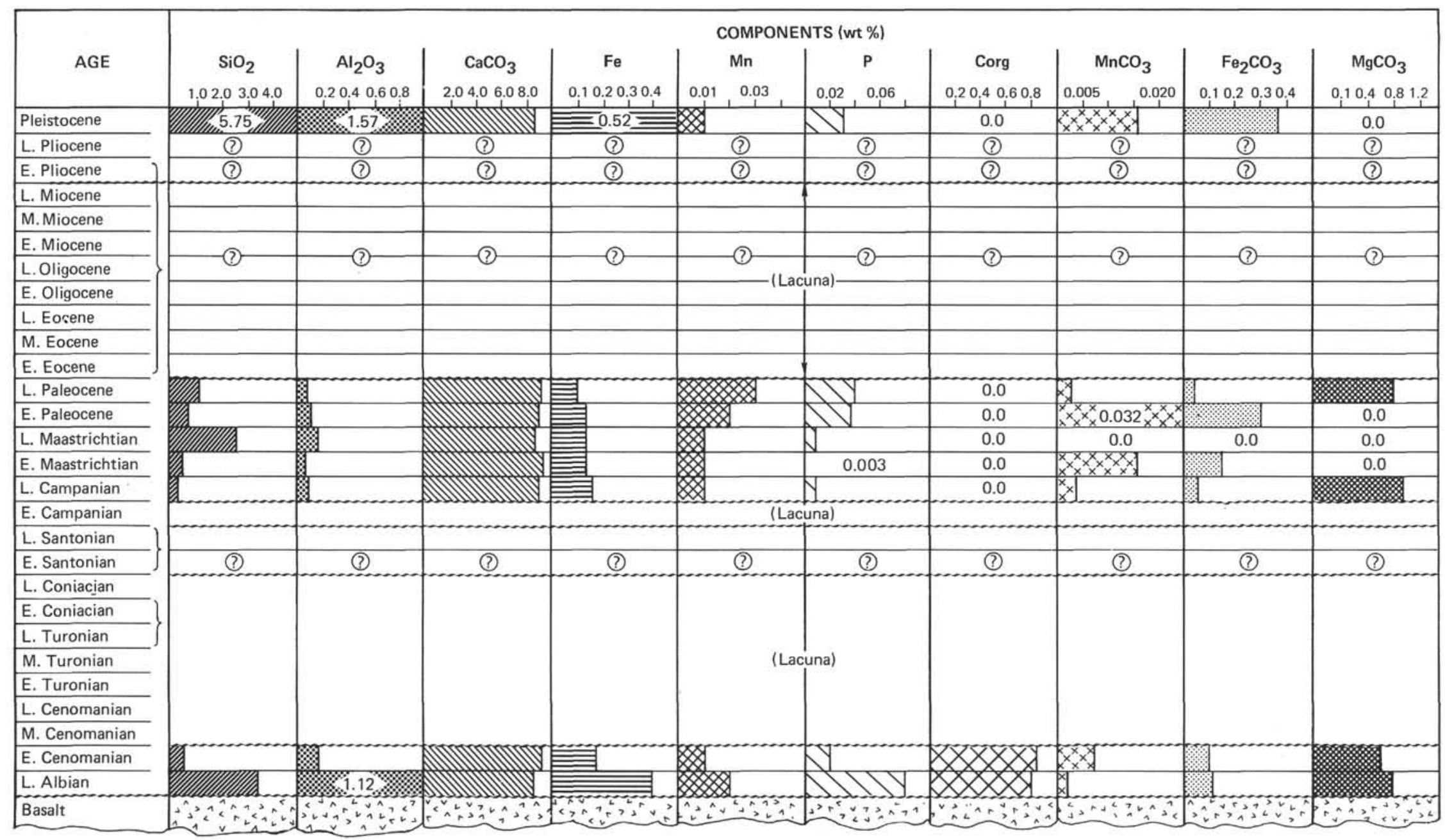

Figure 3. Distribution of average contents (wt. \%, air-dry): $\mathrm{SiO}_{2}, \mathrm{Al}_{2} \mathrm{O}_{3}, \mathrm{Fe}, \mathrm{Mn}, \mathrm{P}$, and normative molecules $\mathrm{CaCO}_{3}, \mathrm{MnCO}_{3}, \mathrm{FeCO}_{3}, \mathrm{MgCO}_{3}$ in a section of post-Jurassic deposits of the central northwestern Pacific, southern Hess Rise, DSDP Site 465. 


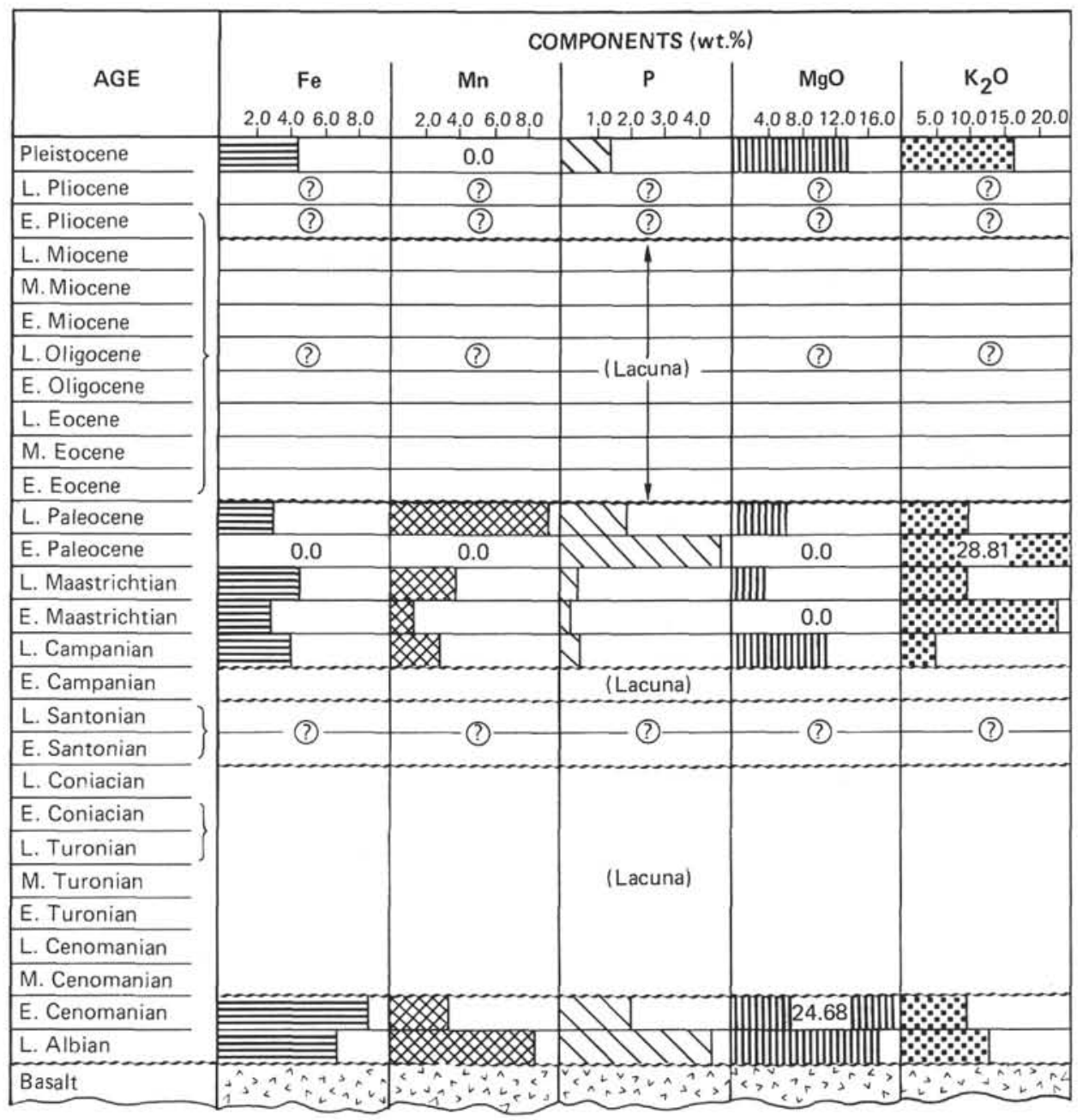

Figure 4. Distribution of average contents (wt. $\%$, recalculated to terrigenous-free, silica-free, carbonate-free) of $\mathrm{Fe}, \mathrm{Mn}, \mathrm{P}, \mathrm{MgO}$, and $\mathrm{K}_{2} \mathrm{O}$ in the section of post-Jurassic deposits of the central northwestern Pacific, southern Hess Rise, DSDP Site 465.

instance, the western Mid-Pacific Mountains, Site 463, Shatsky Rise, Nauru Basin, etc.) of the Pacific Ocean, Atlantic, and Indian Oceans (Schlanger and Jenkyns, 1976; Arthur and Schlanger, 1979) covering various geochronological intervals of the Cretaceous.

Late Cretaceous (middle Cenomanian-late Maastrichtian). The sedimentological significance of an middle Cenomanian-middle Turonian hiatus remains obscure. According to some modifications of the model describing the northward movement of the Pacific Plate (Lancelot and Larson, 1975; Lancelot, 1978; van Andel, 1974), the southern region of Hess Rise during the Cenomanian-Coniacian was in the equatorial zone of high biological productivity. Such a location of the region (if the accepted model proves true, as for many areas of the northwestern Pacific) could have resulted in accumulation of considerable amounts of carbonate sediments. It should be emphasized that during the whole Meso-Cenozoic history of sedimentation the southern part of Hess Rise was situated above the CCD.

It is believed that the development of this hiatus in the region is related to activation of Late Cretaceous analogues of equatorial currents and their northwestern branches (Luyendyk et al., 1972).

During the Santonian, the accumulation of nannofossil and foraminifer-nannofossil oozes with an admixture of siliceous components took place. Relatively decreased thicknesses of these sediments and low rates of their accumulation enable us to regard them as residual after the early Campanian hiatus.

An early Campanian hiatus is the highest of the Late Cretaceous hiatuses of similar nature (Fig. 5).

The final phase of Late Cretaceous sedimentation, during which nannofossil and foraminifer-nannofossil oozes accumulated was the late Campanian-late Maastrichtian. These sediments are characterized by contents of major components and heavy metals characteristic of carbonate pelagic varieties. Worthy of attention are rather high rates of sedimentation and accumulation of major components during the late Maastrichtian (Fig. 5; Table 7). As mentioned above, these data evidence that late Maastrichtian sediments were not affected by erosion, and accordingly they adequately reflect the sedimentation rates of that time. At the same time we can 


\begin{tabular}{|c|c|c|c|c|c|c|c|c|}
\hline \multirow{3}{*}{ AGE } & \multicolumn{2}{|c|}{ SEDIMENTS } & \multicolumn{5}{|c|}{ COMPONENTS (mg-cm $\left.-2 \cdot 10^{-3} \cdot \mathrm{yr}^{-1}\right)$} & \multirow{3}{*}{$\begin{array}{c}P \\
0.10 .20 .30 .4\end{array}$} \\
\hline & $\begin{array}{l}\left(\mathrm{mm}-\mathrm{cm}^{-2}\right. \\
\left.10^{-3} \cdot \mathrm{yr}^{-1}\right)\end{array}$ & $\begin{array}{c}\mathrm{mg}-\mathrm{cm}^{-2} \\
\left.10^{-3} \cdot \mathrm{yr}^{-1}\right)\end{array}$ & $\mathrm{CaCO}_{3}$ & $\mathrm{SiO}_{2}$ & $\mathrm{Al}_{2} \mathrm{O}_{3}$ & $\mathrm{Fe}$ & $\mathrm{Mn}$ & \\
\hline & $\begin{array}{llll}5 & 10 & 15 & 20\end{array}$ & 1000,3000 & $1000 \quad 2000$ & 2.04 .06 .08 .0 & $0.5 \quad 1.0 \quad 1.5 \quad 2.0$ & 1.02 .03 .04 .0 & $0.05,0.15$ & \\
\hline Pleistocene & . & & & & & 目 & 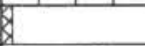 & \\
\hline L. Pliocene & $\therefore$ & 3 & (?) & (?) & (?) & (2) & (2) & (?) \\
\hline E. Pliocene & (?) & (?) & (2) & (2) & (7) & (2) & (?) & (?) \\
\hline \multicolumn{9}{|l|}{ L. Miocene } \\
\hline \multicolumn{9}{|l|}{ M. Miocene } \\
\hline \multicolumn{9}{|l|}{ E. Miocene } \\
\hline L. Oligocene & (2) & (2) & (?) & (7) & (7) & (2) & (2) & (7) \\
\hline \multicolumn{9}{|l|}{ E. Oligocene } \\
\hline \multicolumn{9}{|l|}{ L. Eocene } \\
\hline \multicolumn{9}{|l|}{ M. Eocene } \\
\hline \multicolumn{9}{|l|}{ E. Eocene } \\
\hline \multicolumn{9}{|l|}{ L. Paleocene } \\
\hline \multicolumn{9}{|l|}{ E. Paleocene } \\
\hline L. Maastrichtian & $\because \because \because$ & & & $54.0 \mathrm{Y}$ & $3.3 \%$ & 言 & 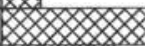 & y \\
\hline \multicolumn{9}{|l|}{ E. Maastrichtian } \\
\hline \multicolumn{9}{|l|}{ L. Campanian } \\
\hline E. Campanian & & & & \multicolumn{2}{|c|}{ (Lacuna) } & & & \\
\hline \multicolumn{9}{|l|}{ L. Santonian } \\
\hline \multicolumn{9}{|l|}{ E. Santonian } \\
\hline \multicolumn{9}{|l|}{ L. Coniacian } \\
\hline \multicolumn{9}{|l|}{ E. Coniacian } \\
\hline \multicolumn{9}{|l|}{ L. Turonian } \\
\hline \multicolumn{9}{|l|}{ M. Turonian } \\
\hline \multicolumn{9}{|l|}{ E. Turonian } \\
\hline \multicolumn{9}{|l|}{ L. Cenomanian } \\
\hline \multicolumn{9}{|l|}{ M. Cenomanian } \\
\hline \multicolumn{9}{|l|}{ E. Cenomanian } \\
\hline L. Albian & 30.9 & 66467 & 5695 & $231.5 \mathrm{Z}$ & 2.42 & 97 & & 5.17 \\
\hline Basalt & 43 & & & 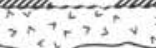 & & $x^{2}+25350$ & 3 & 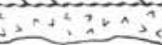 \\
\hline
\end{tabular}

Figure 5. Distribution of average rates of sedimentation and accumulation of components in a section of post-Jurassic deposits of the central north-western Pacific, southern Hess Rise, DSDP Site 465.

assume that during the late Maastrichtian the southern region of Hess Rise had not yet passed across the northern boundary of the sub-equatorial zone of high biological productivity. Only in the latest Maastrichtian was the region displaced into the northern ologotrophic zone of the Pacific Ocean. However, the relatively increased thickness (rate of sedimentation) of the Maastrichtian deposits may also have been caused to a certain extent by the local supply and redeposition of sediments from blocks upraised at this time.

Early Tertiary (early-late Paleocene). The beginning of the Tertiary was characterized by accumulation of nannofossil oozes, with a small admixture of radiolarians and siliceous remains transformed into chert nodules. The composition of major components and heavy metals of these deposits differs slightly from that of typical biogenic carbonate pelagic oozes of the open ocean. Worthy of attention are somewhat higher rates of $\mathrm{Mn}$ and $\mathrm{P}$ accumulation (Table 7; Fig. 5), the nature of which can be due to the appreciable amounts of fine volcaniclastic material of basaltic composition. The available geochemical data, as well as data on mineralogy and lithology of these sediments, show that in the region the transition from Mesozoic to Cenozoic was not marked by well-pronounced changes of geochemical parameters of sedimentation.

Tertiary-Quaternary. During the Eocene-Pliocene, the sedimentation phases appeared to alternate with numerous hiatuses. This finally resulted in accumulation of residual redeposited materials, with sharply decreased thickness (Site 465 report, this volume).

During the late Pliocene and Pleistocene, the accumulation of foraminifer-nannofossil oozes with a slight admixture of siliceous remains and basaltic hyalopelitic-silty (up to 10-15\%) material (Pleistocene) took place. The contents of major components and heavy metals in the sediments under study and rates of their accumulation differ slightly from those typical of biogenic carbonate pelagic oozes of the open ocean.

\section{CONCLUSIONS}

Study of specific features of distribution of major components and heavy metals in the section of Mesozoic and Cenozoic deposits of southern Hess Rise (DSDP, Site 465), analysis of forms of their occurrence on the basis of processing of analytical data by the factor-analysis method, interpretation of results of mineralogic-lithologic studies, and evaluation of distribu- 
tion of average contents of components and rates of their accumulation for the major geochronological subdivisions enable us to outline the main stages in the geochemical history of sedimentation of the region.

\section{Late Albian-Early Cenomanian (early oceanic)}

Accumulation of shallow-water carbonate sediments of turbiditic origin took place. The sediments were enriched with sapropelic organic matter, basaltic volcaniclastic material, and to a lesser extent with hydrothermal and exhalation products. Volcanogenic components resulted in relatively high concentrations of $\mathrm{Fe}, \mathrm{Mn}$, $\mathrm{SiO}_{2}, \mathrm{Al}_{2} \mathrm{O}_{3}$, and associated heavy metals, present mostly in the form of basaltic volcaniclastics and material of their post-sedimentary transformations: smectite-illite, $\mathrm{Mn}-\mathrm{Fe}-\mathrm{Mg}$-carbonates. The rates of sedimentation and accumulation of components were maximal for the post-Jurassic history of the region.

\section{Late Cretaceous (middle Cenomanian- late Maastrichtian)}

This stage is characterized by sedimentation, the results of which were considerably destroyed by the major hiatuses (middle Cenomanian-middle Turonian; late Coniacian-early Campanian). According to a model of the northward movement of the Pacific Plate (Lancelot and Larson, 1975; Lancelot, 1978; van Andel, 1974), the region under study could have been situated during the Cenomanian-Coniacian in the zone of high biological productivity. Only relatively high rates of sedimentation and accumulation of components for the late Maastrichtian enable an approximate evaluation of the final phase of sediment formation near the northern boundary of this near-Equator zone. However, the local contribution and redeposition of sedimentary materials from upraised blocks at this time may somewhat increase the values of the sedimentation rate of the Maastrichtian.

On the whole, the sediments of this stage are similar to biogenic carbonate pelagic oozes of the open ocean, when judged by the known characteristics.

\section{Early Tertiary (early-late Paleocene)}

The beginning of the Tertiary was characterized by accumulation of carbonate nannofossil oozes, admixed with siliceous remains. The available geochemical data do not speak of somewhat abrupt changes of geochemical parameters of sedimentation at the MaastrichtianPaleocene boundary.

\section{Tertiary-Quaternary}

In the Eocene-Pliocene, biogenic carbonate sedimentation is interrupted many times by hiatuses. This resulted in accumulation of redeposited residual sediments of much-reduced thickness. In the late Pliocene and Pleistocene, deposition of foraminifer-nannofossil pelagic sediments with normal geochemical characteristics was proceeding.

Thus, in the geochemical history of post-Jurassic sedimentation of southern Hess Rise, four stages are established. They reflect the evolution of sedimentation from the environment of a relatively shallow-water basin (late Albian-early Cenomanian) to the open ocean (Late Cretaceous-Pleistocene). In our previous works on geochemistry of sedimentation in the region of the western Mid-Pacific Mountains (Site 463) and northern Hess Rise (Site 464), geochemically similar stages of post-Jurassic sedimentation were identified. However, their geochronological duration (for the Cretaceous period particularly) differed significantly, a well-pronounced evolutionary tendency being common.

\section{ACKNOWLEDGMENTS}

It is a pleasant duty to express sincere gratitude to my colleagues at the Geological Institute of the U.S.S.R. Academy of Sciences: P. K. Ryabushkin, D. A. Kazimirov, N. I. Kartoshkina, N. Yu. Vlasova, for appreciable help in processing the analytical data in a computer, recalculation of analyses, and preparation of the materials; V. A. Drits, B. A. Sakharov, T. T. Eliseeva, for X-ray structural analysis; D. Ya. Choporov, for careful control of analytical data; P. P. Timofeev and V. I. Koporulin, for the provided materials and discussion of the results in the course of the study; V. S. Zelinsky and K. A. Shchepoinnikova, for assistance in graphic presentation of the material; G. N. Surovtseva and G. V. Kozlovskaya, for translation of the paper.

Critical review of the paper and comments made by N. G. Brodskaya and A. G. Kossowskaya were useful and provided improvement.

\section{REFERENCES}

Arrhenius, G., 1963. Pelagic sediments. In Hill, M. N. (Ed.), The Sea (Vol. 3): New York (Interscience), 655-727.

1967. Deep Sea sedimentation: a critical review of U.S. works. Trans. Am. Geophys. Union, 48:604-631.

Arthur, M. A., and Schlanger, S. O., 1979. Cretaceous Ocean anoxic events as caused factors in development of reef-reservoired giant oil fields. Bull. Am. Assoc. Petrol. Geol., 63:870-885.

Bezrukov, P. L., and Romankevich, E. A., 1970. Rate of sedimentation in Pacific ocean. In Bezrukov, P. L. (Ed.), Pacific Ocean. Sedimentation in Pacific Ocean (Vol. 2): Moscow (Nauka) 288-300.

Bogdanov, Yu. A., and Chekhovskikh, E. M., 1979. Rates of sedimentation and absolute masses. In Smirnov, V. I. (Ed.) Metalliferous Sediments of South-Eastern Pacific Ocean: Moscow (Nauka), p. 280.

Davis, J. C., 1973. Statistics and Data Analysis in Geology: New York (Wiley).

Harman, H. H., 1967. Modern Factor Analysis: Chicago (Univ. of Chicago Press).

Kirkpatrick, J., 1979. Interlaboratory comparison of Leg 46 standards. In Dmitriev, L., Heirtzler, J., et al., Init. Repts. DSDP, 46: Washington (U.S. Govt. Printing Office), 293-297.

Lancelot, Y., 1978. Relations entre évolution sédimentaire et tectonique de la Plaque Pacifique depuis le Crétacé inférieur. Mém. Soc. Geol. France, 57(134):40.

Lancelot, Y., and Larson, R. L., 1975. Sedimentary and tectonic evolution of the north-western Pacific. In Larson, R. L., Moberly, R., et al., Init. Repts. DSDP, 32: Washington (U.S. Govt. Printing Office), 925-939.

Lisitzin, A. P., 1974. Sedimentation in Oceans: Moscow (Nauka). 1978. Processes of Oceanic Sedimentation: Lithology and Geochemistry: Moscow (Nauka).

Luyendyk, B. P., Forsyth, D., and Phillips, J. O., 1972. Experimental approach to the paleocirculation of the oceanic surface water. Geol. Soc. Am. Bull., 83:2649-2664.

MacArthur, J. M., and Elderfield, H., 1977. Metal accumulation rates in sediments from Mid-Indian Oceanic Ridge and Marie Celeste Fracture Zone. Nature, 266:437-439.

Schlanger, S. O., and Jenkyns, H. C., 1976. Cretaceous oceanic anoxic events: causes and consequences. Geol. Mineral. Jahrb., $55: 179-184$. 
Tiercelin, J. J., and Faure, H., 1978. Rates of sedimentation and vertical subsidence in neorifts and paleorifts. Tectonics and Geophysics of Continental Rifts, (Vol. 2): Moscow (Dordrecht), 41-47.

van Andel, Tj. H., 1974. Cenozoic migration of the Pacific Plate, northward shift of the axis of deposition and paleobathymetry of the Central Equatorial Pacific. Geology, 2:507-510.
Varentsov, I. M., and Blazhchishin, A. I., 1976. Ferromanganese nodules. Geology of Baltic Sea: Vilnius (Mokslas), pp. 307-348.

Zolotarev, B. P., and Choporov, D. Ya., 1978. Petrochemistry of basalts D/V Glomar Challenger, Leg 45, Holes 395, 395A, 396. In Melson, W. G., Rabinowitz, P. D., et al., Init. Repts. DSDP, 45: Washington (U.S. Govt. Printing Office), 479-492. 